\title{
Psychopathologie des Eunuchoidismus und dessen Beziehungen zur Epilepsie.
}

\author{
Von \\ Dr. Heinrich Fischer, \\ Assistenzarzt an der psychiatrischen Klinik in Gießen. \\ Mit 6 Textabbildungen. \\ (Eingegangen am 5. April 1919.)
}

Die Kenntnis der normalen Formen und Proportionen im äußeren Habitus des Menschen ist für den Arzt von hervorragender Bedeutung. Nur so ist es ihm möglich, typische und atypische Abänderungen der äußeren Körperformen gut zu erfassen und in den Krankengeschichten zu beschreiben. Von welcher Wichtigkeit eine solche Beschreibung auch später noch sein kann, lehrt uns die Beschäftigung mit der Literatur über den Eunuchoidismus. Daß sich bei Bildungsfehlern im Sexualsystem auch sonstige den Degenerationszeichen zugerechnete Anomalien fanden, war eine altbekannte Tatsache. So können wir noch heute in der älteren Literatur aus der Beschreibung solcher Anomalien die Zugehörigkeit des Falles zum Eunuchoidismus erkennen. Auch in älteren Krankengeschichten gibt uns die Notiz des einen oder anderen im beschriebenen Falle besonders hervorstechenden Symptomes des Eunuchoidismus unter dem Sammelsurium der Degenerationszeichen Anhaltspunkte. Daß alle diese beschriebenen Anomalien, Degenerationszeichen und Symptombilder etwas Einheitliches darstellen und eine gemeinsame Ätiologie in der Hypoplasie der Geschlechtsdrüse besitzen, lehrte die verdienstvolle, Arbeit von Tandler und Gro $B^{1}$ ).

Die körperlichen Symptome des Eunuchoidismus sind gleich denen, wie sie nach der Kastration aufzutreten pflegen, und wie der Name sagt, gibt uns die Konstitutionsanomalie des Eunuchoidismus eine natürliche Nachahmung des Eunuchen.

Die Kastration ist in ihren Bedingungen ein durchaus differentes Experiment, je nachdem sie vor oder nach der Pubertät ausgeführt wird, und demnach auch in ihrem Resultat. Sie ist um so eingreifender, je jünger das kastrierte Individuum ist, und die präpuberale und

1) Tandler und Grosz, Wiener klin. Wochenschr. 1907; Archiv f. Entwicklungsmechanik 27, 35. 1909; 30, 235. 1910; 29, 290. 1910; Wiener med. Wochenschrift 63, 1409. 1913. 
postpuberale Kastration sind scharf zu trennen. Unter den vor der Pubertät Kastrierten, wie unter den Eunuchoiden unterscheidet man im allgemeinen zwei Typen, die hochwüchsigen, die erfahrungsgemäß häufigeren, und die kleineren fettwüchsigen Individuen. Diese Einteilung hat natürlich nur insoweit Berechtigung, als sie uns die beiden möglichen Grenzbilder gibt, zwischen denen alle graduellen Utbergänge mit Ausprägung der charakteristischen Disproportion des Skeletts und der Charakteristica an den äußeren Bedeckungen möglich sind. Der Späteunuchoidismus liefert uns Bilder, wie sie die Spätkastration liefert.

Während die Folgen der Frühkastration männlicher Individuen von alters her bekannt sind, haben wir über die Frühkastration weiblicher Individuen keine eingehenden Kenntnisse. In der Hauptsache wohl aus äußeren Gründen. Einmal wegen der Schwierigkeit der Operation, und zweitens wegen dem Mangel an Nachfrage. Dagegen scheint die konstitutionelle Hypoplasie der Geschlechtsdrüse beim weiblichen Geschlecht nicht viel seltener zu sein als beim männlichen. Da der eunuchoide Habitus bei oberflächlicher Betrachtung dem weiblichen ähnlicher wirkt als dem männlichen, ist der Eunuchoidismus beim Weibe weniger augenfällig als beim Manne. Da zudem der verspätete Eintritt der Menstruation und andere sexuelle Ausfallserscheinungen im allgemeinen beim weiblichen Geschlecht nicht unangenehm empfunden werden, werden solche Fälle auch seltener dem Arzte zu Gesicht kommen. Die Hypoplasie der weiblichen Genitalien finden wir in der gynäkologischen Literatur häufig als Infantilismus beschrieben. Ein gutes Kriterium für den Beginn der Geschlechtsreife bietet uns beim weiblichen Geschlechte der Eintritt der Menstruation. Wollten wir uns aber nur bei der Diagnose an das Ausbleiben der Menstruation und die Hypoplasie des Genitalapparates halten, so würden wir auch irrtümlich die hypoplastische Konstitutionsanomalie in das Material der Betrachtung einbeziehen, wie umgekehrt nach meinem Eindruck aus der entsprechenden Literatur von gynäkologischer Seite die eunuchoide. Konstitution unter die hypoplastische gerechnet ist. Noch mehr als beim Manne sind wir beim weiblichen Eunuchoidismus auf eine genaue Körperbeschreibung und besonders auf die Körpermaße angewiesen, da die übrigen Symptome des Eunuchoidismus an den äußeren Bedeckungen, Fettverteilung und Behaarung beim weiblichen und eunuchoiden Menschen große Ähnlichkeit aufweisen. Die hypoplastische Konstitution und der Infantilismus müssen unbedingt auch infantile resp. juvenile Wachstumsverhältnisse aufweisen. Schon die Einteilung. des Infantilismus beim weiblichen Geschlecht in verschiedene Formen, eine thyreogene, hypophysäre und genitale Form, einen partiellen und universellen, ja sogar lokalen Infantilismus muß den $\mathrm{Zweifel}$ an 
eine kritikvolle Abtrennung aufkommen lassen. Man hat alles Infantilismus genannt, was von der Form des Erwachsenen abweicht, jede Konstitutionsanomalie, auch wenn sie mit kindlichen Dimensionen und Proportionen nichts gemeinsam hat. Ja man hat sogar den Begriff Infantilismus auf Folgezustände nach überstandenen Krankheiten ausgedehnt. Es sind daher von gynäkologischer Seite beschriebene psychische Veränderungen solcher Individuen nur schwer zu verwerten, da hier zuviel unter einen Begriff gebracht ist. Jedenfalls muß man bei verspätetem Eintritt der Menstruation, Dysmenorrhöe, mangelhaftem Sexualtrieb, Kohabitationsbeschwerden, engem Becken, Sterilität usw. auch an den Eunuchoidismus denken.

Am augenfälligsten sind beim Eunuchoidismus die Erscheinungen an den dem Menschen Gestalt gebenden Organsystemen, einmal dem Skelett (die charakteristische Disproportion) und die Folgezustände an den äußeren Bedeckungen (die charakteristische Fettverteilung und Behaarung). Diese Veränderungen prägen einen in der äußeren Form gut charakterisierten Typ.

Das im ganzen grazile Skelett zeigt in den Extremitäten ein gesteigertes Längenwachstum. Hierdurch kommt es zu der Disproportion in den Skelettmaßen, dem Mißverhältnis der zu langen und zu stark entwickelten Extremitäten zu dem kleinen und in den Schultern schmalen Rumpfskelett, und zum Utberragen der Unterlänge über die Oberlänge. Weitere Skelettmerkmale sind: länglicher Schädel, breite Jochbeine, tiefe Nasenwurzel, in seinen Seitenteilen stark entwickelter breiter Unterkiefer, steiles Hinterhaupt, wenig vorspringende Prominentia laryngea. Das Becken erscheint breit, geringe Lordose der Lendenwirbelsäule, Genu-valgum-Stellung der Beine. Dazu kommt eine charakteristische Fettverteilung bei verschieden großer Fettentwicklung. Fettansammlungen finden sich an der Brust, Unterbauchgegend, Mons pubis, Cristae und Außenseite der Oberschenkel. Die Körperbehaarung ist spärlich, mangelnder Bartwuchs, an seiner Stelle meist eine mäßige Lanugobehaarung, spät einsetzende spärliche Behaarung der Achselhöhle und der Regio pubis, meist nur an der Peniswurzel, oder nach oben in einer geraden resp. konkaven Linie abschneidend, die Linea alba, Damm- und Analgegend frei lassend. Dagegen ist das Kopfhaar dicht und geht auch im Alter wenig aus.

Diese Erscheinungen zusammen mit einem schlaffen schwerfälligen Gebaren, der eigentümlichen Färbung der Stimme, dem etwas müden, teilnahmlosen Ausdruck des Gesichtes, das schon früh reich an Runzeln und Falten ist, machen den charakteristischen eunuchoiden Habitus aus.

Bei den von Tandler und Groß ausgeführten Sektionen fand sich die Persistenz der Epiphysenfugen über das normale Alter hinaus, abnorm lange unverknöcherte Schädelnähte, Verzögerung in der Ver- 
knöcherung des Kehlkopfes, auffallend breite, lange und tiefe Fossa. hypophysea und dementsprechend eine große Hypophyse. Das Becken zeigt Abweichungen vom Typ des männlichen, die Crista ist dick und an ihr die Epiphysenfuge noch im ganzen Verlaufe nachweisbar. Aber mit einem weiblichen Becken hat das eunuchoide Becken nichts gemeinsam. Es kommt zu einer Entwicklungshemmung resp. Rückbildung des gesamten Genitalapparates, wogegen der uropoetische Apparat ganz unbeeinflußt bleibt.

Nicht selten sind leichtere vorübergehende Formen des Eunuchoidismus, Fälle von abnorm lange verzögertem Eintritt der Geschlechtsreife. Auch nach Eintritt der Geschlechtsreife lassen sich diese Fälle noch deutlich insbesondere an der Disproportion des Skelettes, aber auch an der mäßigen Behaarung des Mons pubis, die gewöhnlich in einer geraden Linie nach oben abschneidet, erkennen. Nach meinen Erfahrungen ist die in diesen Fällen erlangte Geschlechtsreife auch nur selten eine vollwertige, und nicht selten ist der Eintritt der Involution der Geschlechtsdrüse mit ihren Folgen eine unverhältnismäßig frühe, so daß nach vorübergehendem Ausgleich auch die übrigen Erscheinungen des Eunuchoidismus wieder deutlich zu werden pflegen. Man darf natürlich diese Fälle, wenn sie, wie das nicht selten der Fall ist, erst nach der Involution in ärztliche Beobachtung kommen, nicht zum Späteunuchoidismus rechnen. Sie gehören dem echten Eunuchoidismus an, wenn auch nur in graduell feiner Abstufung. Dem formverändernden Einfluß der Spätkastration sind natürlich durch den Schluß der Epiphysenfugen zwischen 16. und 22. Lebensjahre schon von vornherein Grenzen gesetzt, das Zustandekommen der eunuchoiden Skelettmerkmale ist ausgeschlossen. Auch Adipositas wird bei Spätkastraten resp. Späteunuchoiden nicht regelmäßig beobachtet. Dagegen fehlt niemals die Neigung zur eunuchoiden Fettverteilung, auch treten die Rückbildungserscheinungen am Genitalapparat ein, die Haut bekommt einen helleren Ton und glattere Beschaffenheit, das Haupthaar verliert die Neigung zum Ausfallen, wogegen die übrige Körperbehaarung besonders deutlich bei dem Manne zu schwinden beginnt, die Stimme nimmt die eunuchoide Färbung an. Der schon ausgebildete cerebrale Geschlechtstrieb verliert sich erst nach und nach im Laufe einiger Jahre, es kommt anfangs noch zu seltenen Erektionen und zur spärlichen Ejaculation eines sterilen dünnflüssigen Sekretes aus Prostata und Samenblasen.

Bei Frauen hat man über die Folgen der Spätkastration im Gegensatz zur Frühkastration, infolge der etwas reichlichen therapeutischen Ausnützung der Kastration durch die Frauenärzte, genügend Erfahrung sammeln können. Es finden sich auch bei der kastrierten Frau Rückbildungserscheinungen am gesamten übrigen Genitalapparat. Die Nei- 
gung zum Fettansetzen findet sich bei kastrierten Frauen häufiger als im Klimakterium. Pfister schreibt, daß sich bei kastrierten jugendlicheren weiblichen Individuen die Fettzunahme ganz der jugendlichen Form anbequemt, so daß die Operierten eher frischer und jugendlich üppiger aussehen, die Haut wird heller und zarter und das Kopfhaar üppiger.

Erwähnt sei noch, daß das Vorkommen mehrerer Fälle von Eunuchoidismus in einer Familie nicht selten zu sein scheint, und auch die Heredität eine Rolle spielt.

Die in folgendem beschriebenen Fälle sind aus einem umfangreicheren Material ausgesucht, das ich im Verlauf von 4 Jahren gesammelt habe. Ich habe natürlich nur solche Fälle gewählt, die ganz reine eunuchoide Symptome zeigen. Bei einer Arbeit, die sich mit der noch ungeklärten Frage der krankhaften Symptome in den psychischen Äußerungen des Eunuchoidismus befaßt, ist es natürlich Voraussetzung, daß an der Auswahl der vorhandenen Fälle strenge Kritik geübt wird. Die bekannten körperlichen Symptome des Eunuchoidismus müssen voll ausgebildet sein. Hierher gehören an erster Stelle die bekannten Skelettsymptome, die allein für einen Früheunuchoidismus beweisend sind, dann die Symptome an den äußeren Bedeckungen, die aber auch die Späteunuchoiden in derselben Weise aufweisen und zum Teil den Symptomen bei der Dystrophia adiposogenitalis ähnlich sind, und leicht zur Verwechselung mit letzterer Anlaß geben können. Fälle mit starker Adipositas, die auf eine stärkere Beteiligung des Hypophysenmittellappens schließen läßt, und mit anderen für die Dystrophia adiposo-genitalis charakteristischen Störungen der Haut und ihrer Anhänge (Kopfhaar, Augenbrauen, Wimpern) sind auszuschalten, ebenso solche Fälle, die eine sichtbare Beteiligung der Schilddrüse in Hautveränderungen oder auch z. B. durch cariöse Zähne zeigen. Auch für die pathogenetische Forschung ist eine solche Trennung sehr wesentlich. Man würde anderenfalls zweifellos verleitet sein, z. B. die Schilddrüse von vornherein bei der Ätiologie der psychischen Erscheinungen im wesentlichen in Anspruch zu nehmen, da ja von ihr ein zweifellos großer Einfluß auf das Gehirnwachstum bekannt ist. Wenn man aber sieht, daß auch echte Eunuchoide, bei denen eine Schilddrüseninsuffizienz in keiner Weise hervortritt, dieselben psychischen Erscheinungen des Eunuchoidismus zeigen, so kann man auf einen derartigen Fehler nicht verfallen.

Auch die Einteilung der Eunuchoiden in verschiedene Typen nach den Befunden auf psychopathologischem Gebiete, wie wir sie in der Literatur finden, glaube ich dem Umstande zuschreiben zu können, daß man bei der Auswahl der Fälle nicht vorsichtig genug gewesen ist. 
Weiterhin habe ich bei der Auswahl der Fälle, deren es dem sonstigen Rahmen der Arbeit entsprechend nicht zu viel werden sollten, einmal den verschiedenen körperlichen Typen des Eunuchoidismus Rechnung getragen und andererseits Leute verschiedener Altersstufen und verschiedener sozialer Stellung gewählt. Meine weiteren Ausführungen stützen sich also nicht nur auf das kleine Material der 6 beschriebenen Fälle. Kranke ohne die eunuchoide Disproportion sind, wenn es sich nicht um echten Späteunuchoidismus handelt, keine Eunuchoiden. Hypogenitalismus und Eunuchoidismus sind etwas durchaus Differentes. Letzterer ist ein wohl umschriebenes konstitutionelles Krankheitsbild. Hypogenitalismus dagegen ist nur ein Symptom, das auch z. B. beim Infantilismus, der hypoplastischen Konstitionsanomalie und polyglandulärer Insuffizienz, wie auch verschiedenen Formen des Zwergwuchses vorkommt. Es fehlt aber in diesen Fällen die eunuchoide Disproportion des Skeletts, dagegen sind die Erscheinungen an den Epiphysenlinien vorhanden.

Diese Tatsache ist auch bei Betrachtungen über die Ätiologie des Eunuchoidismus wohl zu beachten.

Fall 1. Landwirtschaftlicher Arbeiter, geb. 28. X. 1875. Am 29. IX. 1915 der Klinik auf einige Tage zur Beobachtung überwiesen. „Infolge der Mißbildung seiner Geschlechtsteile. und seiner geistigen Fähigkeiten" bestanden Bedenken, ihn in das Heer, einzustellen.

Wichtigste anamnestische Daten: Stammt von gesunden Eltern. Fünf Schwestern und zwei Brüder leben. Vier Geschwister verheiratet, haben Kinder. Die unverheirateten und er selbst sind die längsten Mitglieder der Familie. Als Kind sei er nicht größer gewesen als andere Knaben seines Alters. Im 18. Lebensjahre sei er ,stark in die Höhe geschossen ". Um diese Zeit litt er viel an Kopfschmerzen. Mit 20 Jahren sei er $1,75 \mathrm{~cm}$ groß gewesen. Hat nicht aktiv gedient. In der Dorfschule Durchschnittsschüler. War immer gern für sich allein, hatte niemals Freunde. Seit dem 14. Lebensjahr Bauernknecht. Aus den Mädchen habe er sich nie etwas gemacht. Im 18. Lebensjahre sei es ihm aufgefallen, daß sein Geschlechtsteil viel kleiner sei als bei anderen jungen Leuten seines Alters. Gearbeitet habe er immer gern, nur müsse man ihn bei der Arbeit gewähren lassen; wenn jemand dazwischen rede, werde er leicht gereizt. Im allgemeinen sei man mit ihm zufrieden gewesen. $\mathrm{Da}$ er fleißig sei, habe er nicht häufig die Stelle gewechselt. Sonntags gehe er regelmäßig in die Kirche. In seiner freien Zeit spiele er hin und wieder Karten. Er trinke auch gern Bier, könne aber nicht viel vertragen. Aus den Akten sei noch hinzugefügt, daß Pat. sich der Musterung entzog und sich in letzter Zeit vagabundierend umhertrieb. Er ist vielfach wegen Körperverletzung vorbestraft.

Graziler Knochenbau. Enormes Mißverhältnis zwischen dem kurzen schmalen Rumpf und den langen massigen Extremitäten, ungewöhnlich ausgesprochene eunuchoide Disproportion. Überragen der Unterlänge über die Oberlänge. Lünnglicher Schädel, kleiner Kopfschädel, großer und langgestreckter Gesichtsschädel, breite Jochbeine, massiger Unterkiefer, tiefgesattelte Nase, steiles Hinterhaupt, flacher Gaumen. Die Raphe springt im Bereiche des harten Gaumens breit und wulstartig vor. Prominentia laryngea auch bei Schluckbewegungen nicht sichtbar und nur wenig zu tasten. Schilddrüse tastbar. Im Verhältnis zu dem schmalen Schultergürtel breiter Beckengürtel, geringe Lordose der Lendenwirbelsäule, leichte Genu-valgum-Stellung der Beine, flaches Fußgewölbe. 
Schlechtes Fettpolster, trotzdem noch deutliche eunuchoide Fettverteilung an der Brust, Hüften, Unterbauchgegend und Mons pubis.

Kopfhaar dicht. An Stelle des Bartes eine leichte Lanugobehaarung. Auch in der Achselhöhle etwas Lanugobehaarung und einige stärkere Haare. Schamhaare nur in der Ungebung der Peniswurzel. Die Muskulatur des Schulter- und Beckengürtels ist im Verhältnis zu der der Extremitäten schlecht entwickelt. Die Muskulatur zeigt kaum vorspringende Form, der Tonus ist schwach. Die distalen Enden der Extremitäten, besonders der oberen, sind stark livide verfärbt. Der Penis ist sehr klein, $21 / 2 \mathrm{~cm}$ lang. Das Scrotum ist klein, flach ausgespannt. Der linke Hoden etwa bohnengroß, derb, im Scrotum fühlbar. Rechts Kryptorchismus, Leistenkanal offen.

Körpermaße: Größe $181 \mathrm{~cm}$, Spannweite $202 \mathrm{~cm}$. Die Mitte des Körpers, die beim erwachsenen Mann am unteren Rand der Schambeinfuge liegen soll, liegt bei Pat. noch etwas unterhalb des Dammes, es überragt also die Unterlänge die Oberlänge um mehrere Zentimeter. Das Bein von der Spina iliaca ant. sup. bis zum Malleolus internus mißt $103 \mathrm{~cm}$. Die Armlänge (Humeruskopf bis Spitze des Mittelfingers) $89 \mathrm{~cm}$. Die Armlänge soll in der gesamten Körperlänge 2,5 mal enthalten sein, sie ist im vorliegendem Falle nur 2,3 mal in der Körperlänge enthalten. Brustumfang $95,5 \mathrm{~cm}$, Hüftenumfang $96 \mathrm{~cm}$. Schädelumfang $56 \mathrm{~cm}$.

Röntgenologische Befunde: Sella groß, Nebenhöhlen, besonders Kejlbeinhöhle, groß. Unterkiefer massig. Epiphysenfugen am sternalen Clavicularende, am proximalen Humerusende, am distalen Ende von Radius und Ulna, am proximalen Ende der Tibia und Fibula unverknöchert.

Urin Tagesmenge: $1800-3000 \mathrm{ccm}$, spez. Gew. 1010-1020. $\mathrm{E}+\mathrm{Z}$ negativ.

Alimentäre Glykosurie bei $100 \_400 \mathrm{~g}$ Trauben-

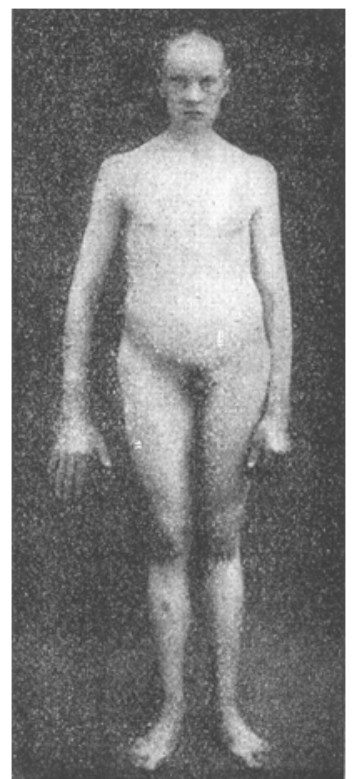

Abb. 1. zucker negativ. Nach $1 \mathrm{ccm}$ Suprarenin Urinmenge 2000, spez. Gew. 1010. Z. negativ. Als Allgemeinsymptom tritt nach Suprarenin ein rhythmisches Zittern des ganzen Körpers und Blässe des Gesichtes ein.

Blutuntersuchung: Erythrocyten 5000000 , Hämoglobin $90 \%$, Leukocyten: 5600 , davon Neutrophile $68 \%$, Eosinophile $3 \%$, Lymphozyten $20 \%$, Mononucleäre und Übergangsformen 9\%, Mastzellen 1\%. Die Körpertemperatur ist normal.

Blutdruck $132 \mathrm{~mm} \mathrm{Hg}$, steigt nach Suprarenin innerhalb 25 Minuten gleichmäßig auf 145 an und wird nach weiteren 20 Minuten wieder normal. Unmittelbar nach der Injektion war er auf 128 gefallen. Nach der Injektion Herzklopfen, Beklemmungsgefuhl und heftiges Zittern. Nach Pilocarpin 0.01 etwas Schweißausbruch, keine Salivation. Nach Phloridzin subcutan Zucker im. Urin positiv.

Befund an den inneren Organen und neurologischer Befund: O. B.

Stimmlage hoch mit der den Eunuchoiden eigentümlichen Färbung. Eine Vita sexualis fehlt völlig.

Das Gesicht zeigt den Ausdruck der Ermidung und des Erstaunens. Dieser Gesichtsausdruck ist der ständige, die Mimik ist sehr ärmlich, dazu verschärfen die vielen Fältchen und Runzeln, sowie die tiefen Querfalten auf der Stirn den Eindruck des Gleichförmigen. Haltung und Gang sind unelastisch, schwerfällig, eine 
schlaffe Müdigkeit liegt in dem ganzen Gebaren des Pat. Seine Antworten erfolgen langsam und brockenweise, er spricht nicht in zusammenhängenden Sätzen. Urteilsfragen werden, sobald sie den engen Rahmen seines Lebenskreises und seiner Interessen überschreiten, flach und dürftig beantwortet. Bei einer Prüfung seiner Kenntnisse zeigt sich, daß diese kaum dem Durchschnitt selbst von Leuten seiner sozialen Stellung entsprechen. So könnte es bei oberflächlicher Prüfung den Anschein haben, als handle es sich um einen Schwachsinn leichten Grades. Bei eingehender Beschäftigung mit ihm zeigt sich aber, daß kein Intelligenzdefekt vorliegt, daß man sich aber zunächst durch seine große Faulheit und Trägheit hindurcharbeiten $\mathrm{mu}$, um zu richtigen Resultaten zu gelangen. Man sieht dann, daß seine intellektuellen Fähigkeiten gar nicht schlecht sind. Sein Mangel an Initiative, seine Denkfaulheit und Trägheit sind es auch, die ihn vor einem größeren geistigen Erwerb geschützt haben.

Er ist schwer zugänglich, mißtrauisch, abweisend, teilnahmlos und untätig. Während der Untersuchungen brauste er einige Male reizbar auf, „da man ihn mit den ewigen Fragen quäle". Die Fähigkeit sozialer Einfühlung ist bei ihm nur gering ausgebildet und gehemmt durch eine starke egozentrische Einengung. Auch in das Leben auf der Abteilung fügte er sich nicht ein, sondern stand außerhalb. Für die Verwerflichkeit seines Verbrechens (Fahnenflucht) fehlte ihm das Verständnis.

Fall 1 ist nach den körperlichen Symptomen ein Fall von reinem eunuchoiden Hochwuchs. Vita sexualis hat niemals, auch nicht in Andeutungen bestanden. Patient ist körperlich und geistig träge und schwerfällig. Seine Interessenlosigkeit und Denkfaulheit zusammen mit einem mangelhaften erworbenen geistigen Besitzstand, dessen Lückenhaftigkeit wieder auf die angeführten' Eigenschaften zurückzuführen ist, täuschen bei oberflächlicher Betrachtung einen Schwachsinn leichteren Grades vor. Bei eingehender Untersuchung zeigt sich, daß seine intellektuellen Fähigkeiten dem Durchschnitt entsprechen. Er ist schwer zugänglich, abweisend, mißtrauisch, teilnahmlos, untätig, egozentrisch eingeengt. Hochwertige soziale Gefühle fehlen. Er ist reizbar und alkoholintolerant, schon nach kleineren Mengen steigert sich seine Reizbarkeit derartig, daß er mit dem Gesetz in Konflikt gerät, und diesem Umstande sind auch nach seiner Angabe die vielfachen Vorstrafen wegen Körperverletzung zuzuschreiben.

Fall 2. Waschmeister, 45 Jahre alt.

Zur Vorgeschichte: Angeblich gesunde Eltern. Sein Stiefbruder war hochgradig schwachsinnig. Erste Entwicklung des Pat. verspätet. Im zweiten Lebensjahr schwere fieberhafte Erkrankung. Er war ein stilles, artiges, schüchternes Kind. In der Schulzeit hat er niemals Freundschaft geschlossen, war lieber für sich allein. Mittelmäßiger Schüler. Vom Militär wegen X-Beinstellung frei. Seit dem 16. Lebensjahre wisse er, daß er geschlechtlich nicht so wie andere Männer entwickelt sei. Sein Geschlechtsteil sei seit dieser Zeit nicht mehr gewachsen. Er habe sich niemals um das weibliche Geschlecht gekümmert, am Tanzen und am Sport habe er keine Freude gehabt. Er habe sich immer schwer angeschlossen und niemals einen Freund gehabt. In den Jugendjahren habe er viel an Kopfschmerzen gelitten und sei fetter geworden. Im 39. Lebensjahre erlitt er eine Gehirnerschütterung. Seine Leistungsfähigkeit habe seit dem Unfall erheblich nachgelassen und er leide oft unter Ver- 
s timmungen, Kopfschmerzen, Schwindelanfällen und Angstanfällen. Manche T age habe er ein Gefühl von Ameisenlaufen und Jucken am ganzen Körper.

Stark ausgesprochene eunuchoide Disproportion des Skeletts, länglicher Schädel, breite Jochbeine, kräftiger Unterkiefer, steiles Hinterhaupt. Die Raphe des harten Gaumens springt wulstartig vor. Prominentia laryngea nicht sichtbar, auch nur wenig zu tasten. Beckengürtel im Verhältnis zum Schultergürtel zu breit, geringe Lordose der Lendenwirbelsäule, Genu-valgum-Stellung der Beine, flaches Fußgewölbe. Fettpolster gut entwickelt bei ausgesprochener eunuchoider Fettverteilung. Starke'Fettansammlungen an den Brüsten, Hüften und Unterbauchgegend, die durch eine tiefe Einziehung von dem noch fettreicheren, ungewöhnlich stark vorspringenden Mons pubis abgesetzt ist, und an der Außenseite der Oberschenkel. Kopfhaar gut entwickelt. An Stelle des Bartes und der Behaarung der Achselhöhle leichte Lanugobehaarung. Einige wenige Schamhaare an der Peniswurzel. Die Haut hat ein zartes, pastöses Aussehen. Muskulatur mäßig kräftig, die Formen weich und rund, ohne vorspringende Muskelwülste, Tonus der Muskulatur schwach. Distale Enden der Extremitäten livide. Penis sehr klein, in dem vom Mons pubis kaum abgesetztem Scrotum zwei kleine derbe Knoten.

Körpermaße: Größe $165 \mathrm{~cm}$, Spannweite $186 \mathrm{~cm}$, Gewicht 65,4 kg. Die Mitte des Körpers liegt anstatt am unteren Ende der Schambeinfuge in Höhe des Dammes, es überragt also die Unterlänge die Oberlänge. Armlänge $77 \mathrm{~cm}$, in der Körperlänge anstatt 2,5 mal nur 2,1 mal enthalten.

Röntgenologischer Befund: Normale Sella. Von den Epiphysenfugen wurden nur die am distalen Ende von Radius und Ulna, Hand und am proximalen Ende von Tibia und Fibula nachgesehen, sie waren sehr schmal, aber Verknöcherung war nur stellenweise vorhanden. An den Phalangen der Hand fanden sich sog. Compactainseln, und zwar strich-

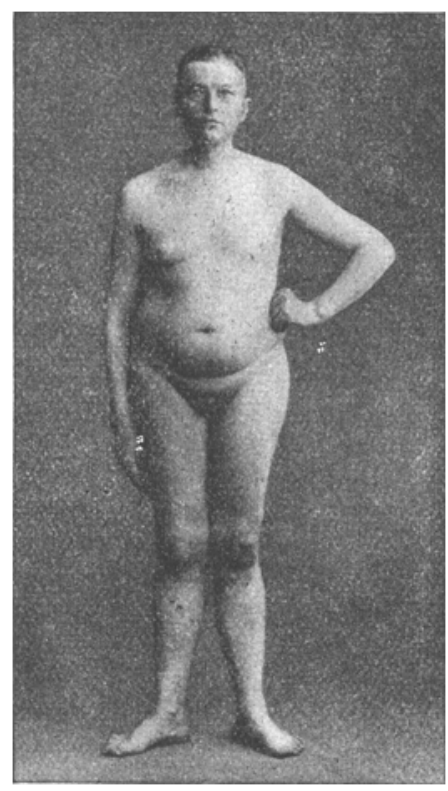

Abb. 2. förmig, an den proximalen Enden der Grundphalangen besonders deutlich, und zwar in Höhe der Epiphysenlinien. Urintagesmenge: 2000-2500, spez. Gew. 1020. Alimentäre Glykosurie bis $400 \mathrm{~g}$ Traubenzucker negativ. Untersuchung der inneren Organe und neurologische Untersuchung zeigen normalen Befund. Körpertemperatur 35,6-36,2 ${ }^{\circ}$. Der Puls ist klein, von guter Spannung, gleichmäßig und regelmäßig. Der Blutdruck beträgt $140 \mathrm{~mm} \mathrm{Hg}$. Nach $1 / 2 \mathrm{ccm}$ Suprarenin steigt der Blutdruck um' $10 \mathrm{~mm} \mathrm{Hg}$ und als Allgemeinreaktion tritt Blässe und Zittern ein.

Stimmlage hoch mit eunuchoider Färbung. Niemals Erektionen, keine Vita sexualis. Das Gesicht ist reich an Falten und Runzeln, die Mimik ist arm, trotzdem verleihen die kleinen lebhaften Augen dem Gesicht einen intelligenten Ausdruck. Haltung und Bewegung unelastisch und sohwerfällig. Pat. machte einen gedrückten apathischen Eindruck. Er zeigte sich zurückhaltend und"mißtrauisch, Interesse für seine Umgebung zeigte er nicht, auch beschäftigte er sich nicht. Bei der Verfechtung seiner Rentenansprüche (es handelte sich um eine Rentennachprüfung) 
zeigte Pat. eine gewisse Schlauheit. Ob auch schon vor dem Unfall seine jetzigen Beschwerden, Verstimmungen mit Kopfschmerzen, kurze Schwindelanfälle und Angstanfälle bestanden hatten, war nicht festzustellen, da er alles auf den Unfall schob. Er erklärte, es sei ihm am angenehmsten, wenn die Untersuchung so ausfiele, daß er keine Arbeit mehr zu verrichten brauche. Der Vorhalt, daß er ein durchaus arbeitsfähiger Mensch in den besten Jahren sei, löste bei ihm gar keinen Affekt aus. Störungen der intellektuellen Fähigkeiten bestanden nicht, doch bestand eine deutliche Verlangsamung und Erschwerung in seinen Reaktionen.

Fall 2 ist nach der körperlichen Untersuchung ein fettwüchsiger Eunuchoider. Er ist träge, schwerfällig, zurückhaltend, mißtrauisch, teilnahmlos, untätig, gedrückt. Die Intelligenz ist normal, es besteht eine Verlangsamung in den Reaktionen. Seit den Jünglingsjahren leidet er an Kopfschmerzen. Im Anschluß an einen Unfall traten angeblich zum erstenmal im 39. Lebensjahre Verstimmungen, kurz dauernde Schwindelanfälle und Angstanfälle auf.

Fall 3. Stud. theol, 22 Jahre alt. Beim Vater șoll die Geschlechtsreife ,ziemlich spät" eingetreten sein, leidet an beiderseitiger Katarakt. Die Mutter leidet hin und wieder an Kopfschmerzen. Von den beiden noch im präpuberalen Alter stehenden Brüdern des Pat. litt der eine bis zum 2. Lebensjahre an Krämpfen. Erste Entwicklung des Pat. normal, war immer etwas schwächlich. Nach Angabe der Mutter ein stilles, artiges, schüchternes Kind gewesen, habe schwer Freundschaft geschlossen, war schwer zugänglich, beschäftigte sich allein. Jetzt sei er im allgemeinen ein stiller und gutmütiger Mensch, nur an manchen Tagen recht reizbar. In der Schule kam er gut mit, machte mit 19 Jahren die Reifeprüfung. „Er war mehr zum Grübeln veranlagt und sehr religiös."

Nach seinen Angaben sollen einige Erektionen etwa mit dem 14. Lebensjahre aufgetreten, aber schon nach kurzer Zeit gänzlich wieder geschwunden sein. Bald danach trat gesteigertes Längenwachstum ein. Erst mit dem 20. Lebensjahre traten hin und wieder von neuem Erektionen ein. Anfänge einer Schamhaarbildung fallen in das 15. Lebensjahr, machten aber dann keine weiteren Fortschritte. Die Erektionen sind selten, unvollständig und ohne sexuelle Gefühlsbetonung. Stimmwechsel hat noch nicht eingesetzt. Er pflegt wenig Kameradschaft, trinkt keine alkoholischen Getränke und raucht nur selten. Zu Beginn des Krieges gemustert und zum Train eingezogen, schon nach 8 Tagen ,,wegen zu geringer Entwicklung “" als d. u. entlassen. Bei neuer Musterung wegen „Habitus femininus“ D. u. geschrieben. Bei der nächsten Musterung kriegsverwendungsfähig für Infanterie. Er hielt die Anstrengungen des Garnisondienstes nicht aus. Bei Übungen, die Muskelkraft erforderten, versagte er. Pat. wurde darauf von den Märschen befreit. Als er trotzdem einen Marsch mitmachen mußte, brach er nach der Rückkehr auf dem Kasernenhof ohnmächtig zusammen. Einige Wochen später traten zum erstenmal kurze Schwindelanfälle auf. Die Schwindelanfälle leiten sich ein mit Angstgefühl und einem Gefühl, als ob das Herz langsamer schlage, sie sind an heißen Tagen häufiger als an kalten. In letzter Zeit sind die Anfälle gehäuft aufgetreten, bis zu 4-5 mal am Tage. In der Zeit zwischen den Anfällen fühlt Pat. sich körperlich schlaff und müde. Der Knpf ist dösig. Manchmal hat Pat. hinterher das Gefühl „als sei er selbst ein Schemen", ,auch die Umgebung erscheine schemenhaft unwirklich", der Schlaf sei bisweilen tief und bleiern. Am frischesten fühle er sich morgens, im Laufe des Tages werde der Kopf benommener, gegen Abend sei das Allgemeinbefinden am schlechtesten. Er sei an solchen Tagen, an denen die Anfälle auftreten, schreckhaft, unruhig, reizbar und hastig in den Bewegungen, gehe 
ziellos umher und habe Herzklopfen. Solche Zustände seien bisweilen von 3-5 tägiger Dauer mit Pausen von 3-4 Wochen, in letzter Zeit seien die Pausen immer kürzer geworden.

Graziles Skelett mit ausgesprochenen eunuchoiden Proportionen. Hirnschädel quadratisch, Gesichtsschädel im Verhältnis zum Hirnschädel groß, Jochbeine vorspringend, Unterkiefer massig, Kinn rundlich, wenig vorspringend. Knöcherner Gaumen asymmetrisch. Prominentia laryngea auch beim Schlucken nicht sichtbar und wenig zu tasten. Der Schultergürtel ist schmal. Infolgedessen erscheint der Beckengürtel zu breit. Leichte Genu-valgum-Stellung der Beine, flaches Fußgewölbe. Fettpolster mäßig, aber eunuchoide Verteilung deutlich. Die Haut ist zart und pigmentarm, erweiterte Hautvenen am Nacken und an den Oberschenkeln. Die Muskulatur ist schlaff und mäßig entwickelt, die Formen sind weich und rund. Kopfhaar dicht, Augenbrauen und Wimpern gut. Kein Bartwuchs. Spurenweise Behaarung der Achselhöhlen und der Peniswurzel. Penis klein, $4 \mathrm{~cm}$ lang, Hoden klein und derb, verschieden groß, nur auf starken Druck schmerzhaft. Distale Enden der Extremitäten livide. Im Röntgenbilde ist die Sella groß, die Epiphysenlinien noch nicht verschmolzen. Körpermaße: Körpergröße $170 \mathrm{~cm}, \mathrm{Ge}-$ wicht 56,4 kg. Spannweite $185 \mathrm{~cm}$, Scheitel-Damm $81 \mathrm{~cm}$, es überragt also die Unterlänge die Oberlänge erheblich. Hüftkamm-Fußsohle $111 \mathrm{~cm}$, Trochanter-Fußsohle $98 \mathrm{~cm}$, Armlänge $80,5 \mathrm{~cm}$ (anstatt 2,5 nur 2,1 mal in der Körperlänge enthalten), Hand $19 \mathrm{~cm}$, Fußlänge 26, $5 \mathrm{~cm}$, Fußhöhe $6 \mathrm{~cm}$. Entfernung der Spinae iliac. $27 \mathrm{~cm}$, der Cristae $30 \mathrm{~cm}$, der Trochanteren $33 \mathrm{~cm}$, Kreuzbeinsymphyse $18 \mathrm{~cm}$. Brustumfang $72 / 88 \mathrm{~cm}$.

Urinmenge 1200-1900 ccm, spez. Gew. 1014-1017. Alimentäre Glykosurie geprüft bis $250 \mathrm{~g}$ Traubenzucker negativ. Nach Adrenalin + Cocain Zuckerreaktion negativ. Blutuntersuchung: Rote Blutkörperchen 5100000 , Hämoglobin $85 \%$. Weiße Blutkörperchen 10,200, davon Neutrophile $61 \%$, Eosinophile $9 \%$, Lymphocyten $22 \%$, Mononucleäre und Übergangsformen $8 \%$. Mastzellen wurden nur sehr selten gefunden. Nach $1 \mathrm{ccm}$ Suprarenin stieg der Blutdruck von $135 \mathrm{~mm}$ $\mathrm{Hg}$ innerhalb einer halben Stunde auf $160 \mathrm{Hg}$. Die Körpertemperatur stieg um $1^{\circ}$. Die Pulsfrequenz stieg von 80 auf 120 in der Minute. Schon bald nach der Injektion klagte Pat. über Klopfen im Kopf, Unruhe im Kopf, Tremor der Hände dann später über Herzklopfen und Zittern im ganzen Körper.

Die Körpertemperatur hielt sich beständig um $36^{\circ}$. Nach Hypophysin stieg die Temperatur innerhalb 45 Minuten um $12^{\circ}$, ohne Beeinflussung der Pulsfrequenz. Die neurologische Untefsuchung und die Untersuchung der inneren Organe ergaben normale Befunde.

Ich hatte Gelegenheit, Pat. 1/2 Jahr lang täglich auch im Dienst (er war Sanitätsgefreiter) zu beobachten. Er hat eine gute Intelligenz und ist ein stiller, zurückhaltender Mensch. Bei den Arbeitsleistungen gewissenhaft, aber ohne bemerkenswert unter seiner stumpfsinnigen Arbeit (Abschreiben und Schreiben nach Diktat) zu leiden. Er zeigte wenig Selbständigkeit und Initiative. Er war schwer zugänglich und bei seiner Jugend auffallend resigniert und mißtrauisch. Ohne Ehrgeiz, es drückte ihn nicht, daß er es nicht weiter als bis zum Sanitätsgefreiten bringen werde. Von Anfang an war ihm der Gedanke, sich freiwillig zu melden, gar nicht gekommen. Er hatte niemals Sport getrieben, war körperlich träge und schwerfällig. Er litt darunter, daß der Stimmwechsel nicht einsetzen wollte. Er interessierte sich für theologische und philosophische Fragen, doch zeigte er besonders auf philosophischem Gebiete eine geringe und einseitige Literaturkenntnis. Er war einseitig eingestellt in einer mehr strenggläubigen Richtung.

Verstimmungen mit starken Kopfschmerzen und leichten Schwindelanfällen habe ich mehrere beobachtet. Pat. empfand an solchen Tagen Widerwillen und 
Gleichgültigkeit gegen die Umgebung und jede Beschäftigung, er war verlangsamt, schweigsam und leicht gedrückter Stimmung. Er nahm auf meinen Rat längere Zeit Hypophysistabletten. Nach $3 / 4$ Jahren schrieb er mir, daß er sich jetzt körperlich frischer und tatkräftiger fühle, aber der ersehnte Stimmwechsel sei noch nicht eingetreten. Die Verstimmungen und Kopfschmerzen seien etwas seltener geworden.

Bei Fall 3 traten zuerst im 14. und dann wieder seit dem 20. Lebensjahre vereinzelte Erektionen auf, allerdings frei von sexueller Gefühlsbetonung und Vorstellung. Die Erektionen sind bei ihm das einzige Symptom einer Funktion der Geschlechtsdrüse, es besteht danach immerhin die 'Möglichkeit, daß er vorübergehend eine gewisse sexuelle Reife erreichen wird. Jedenfalls zeigt Patient aber zur Zeit alle Symptome eines ausgesprochenen eunuchoiden Hochwuchses. Als erbliche Belastung sind verspätete Geschlechtsreife beim Vater und Migräneanfälle der Mutter zu bewerten.

Als Kind war Patient still, artig, schüchtern, ängstlich. Er hat eine gute Intelligenz, ist zum Grübeln veranlagt, sehr religiös, dabei auffallend resigniert und mißtrauisch. Bei der Arbeit ist er fleißig, aber stumpfsinnig ohne Selbständigkeit und Ehrgeiz. Er ist schwerfällig, ohne Neigung und Bedürfnis zum AnschluB. Patient litt an reizbaren Verstimmungen, kurzdauernden Schwindelanfällen, Ohnmachten und schwerer Migräne.

Fall 4. Schüler, geb. am 15. V. 1899. Zum erstenmal vom 6. X. 1915 bis 2. XII. 1915 in klinischer Beobachtung. Er kam mit der Diagnose: Dystrophia adiposo-genitalis. Wichtigste anamnestische Daten: Die Mutter litt in früheren Jahren an Migränenanfällen. Die Pubertätsentwicklung beim Vater trat verhältnismäßig spät ein und die Potenz ließ früh nach. Der Vater hat auffallend lange Extremitäten. Die 18 jährige Schwester des Pat. wurde auch in der Klinik untersucht, zeigte nichts Bemerkenswertes. Sie soll sehr lebhaft und begabt sein. Die Mutter hatte zur Zeit der Schwangerschaft keinerlei Störungen. Pat. wurde im 8. Monat geboren, wog 5 Pfd., war sehr schwächlich und soll infolge Unachtsamkeit der Pflegerin eine starke Blutung aus der Nabelschnur gehabt haben. Mit 11/4 Jahren lernte Pat. laufen, sprechen angeblich zur rechten Zeit, die ersten Zähne kamen verspätet. Mit 4 Jahren Röteln, mit 5 Jahren Diphtherie, mit 9 Jahren Masern; etwas später Scharlach. Bezüglich des Längenwachstums ist zu sagen, daß Pat. mit 9 Jahren an Größe die um 2 Jahre ältere Schwester erreichte. Seitdem ist er langsamer gewachsen. Schon seit dem 10. Lebensjahre zeigte er ,etwas mädchenhafte Formen". Er war ein , gut genährtes Kind, trotzdem er niemals viel, in den letzten Jahren sogar schlecht gegessen hat". Der Geschlechtsteil blieb in der Entwicklung zurück. Seit dem 10. Lebensjahr häufig Kopfschmerzen, mußte deswegen wiederholt die Schule versäumen, wurde infolgedessen im 11. Lebensjahr nicht versetzt. Er wurde 1/4 Jahr vom Schulbesuch dispensiert und aufs Land geschickt, erholte sich dort, das Lernen ging besser. Ende 1913 traten die Kopfschmerzen wieder stärker auf. Der Schlaf wurde immer unregelmäßiger und kürzer. Tageweise klagte Pat. über Dösigkeit. Die Kopfschmerzen verschlimmerten sich bei körperlichen Anstrengungẹn und seelischer Erregung und waren durch Arzneimittel nicht zu beeinflussen. Auch keine Besserung nach Entfernung der Wucherungen aus dem Nasen-Rachenraum im 15. Lebensjahre. Eine Verschlimmerung der 
Kopfschmerzen trat regelmäßig im Frühjahr ein. Seit $1 / 2 \mathrm{Jahr}$ bemerkte die Mutter, daß der Geschlechtsteil an Größe zunimmt, gleichzeitig wurden die Kopfschmerzen geringer. Doch hielt diese Besserung nur kurze Zeit an. Der Stuhlgang ist angehalten. Er leidet viel an Blähungen und Aufstoßen und muß viel gähnen. Er klagt oft darüber, daß es ihm zu heiß sei und er zeitweise schwer Luft bekomme. Die letzteren Beschwerden sollen sich bei Aufenthalt an der See gebessert haben. Immer wenig Hunger, Durst- und Schlafbedürfnis. Er will an den Anzügen bemerkt haben, daß die Extremitäten im letzten Jahr bedeutend gewachsen sind. In der Haut spürt er häufig Jucken, er schwitzt leicht bei körperlicher Anstrengung.

In der ersten Kindheit war er still, artig, ängstlich und für sich allein. In seinem Gefühlsleben sei er jetzt noch sehr kindlich. Er zeigte niemals Neigung zu „dummen Jungenstreichen“. Er sei eher etwas weichlich, empfindsam und leicht gekränkt.

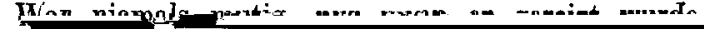
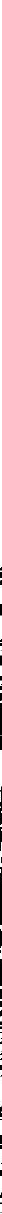

war er niemals der Anführer, ihm fehlten Eh Selbständigkeit, Phantasie und Entschluß] Freundschaft schloß er schwer, gab sich liebes jüngeren Kindern $a b$, unter gleichaltrigen füh sich leicht zurückgesetzt, sie waren ihm zu Trotzdem er nicht ehrgeizig sei, habe er seine $\mathrm{S}$...... arbeiten regelmäßig angefertigt. Er habe ein gutes Gedächtnis, in der Schule gehörte er zu den Durch-
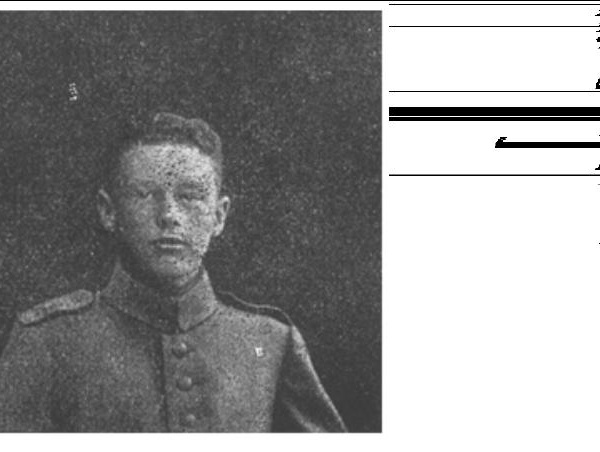

schnittsschülern. Seine Gereiztheit habe in letzter Zeit zugenommen, er neige zu Nörgelei und zur Unzufriedenheit, zum Hinterbringen von allerlei Klatschereien und Angaben. Es fehlen ihm ausgesprochene Neigungen und Interessen. Seit Herbst 1914 wegen Zunahme der Kopfschmerzen und Reizbarkeit kein Schulbesuch mehr. Machte ohne Erfolg eine Liegekur durch. Am 28. VII. 1915 brachte er sich bei einem nicht ganz geklärten Suicidversuch eine Schußverletzung in der rechten Schläfengegend bei, die zu einer Verletzung des Augenhintergrundes und Ptosis des lin- 
ferner übèr den Hüften, an der Außenseite der Oberschenkel und an den Nates. Die distalen Enden der Extremitäten, besonders der unteren sind stark livide verfärbt. Die Haut ist zart, am Stamme leichte Lanugobehaarung, das Kopfhaar dicht und trocken, keine Andeutung von Bartwuchs, Achselhöhlen und Mons pupis ohne Behaarung.

Kör permaße: Körpergröße 162,5 cm, Spannweite $170 \mathrm{~cm}$, Scheitel-Damm $82,5 \mathrm{~cm}$, Hüftbeinkamm-Fußsohle $102 \mathrm{~cm}$, Armlänge 74,5 cm, Handlänge $18 \mathrm{~cm}$. Trochanter-Fußsohle $89 \mathrm{~cm}$, Fußhöhe $5,5 \mathrm{~cm}$, Fußlänge $25 \mathrm{~cm}$, Schulterbreite $35 \mathrm{~cm}$, Brustumfang $86 / 90 \mathrm{~cm}$, Umfang über den Hüften $70 \mathrm{~cm}$, Umfang der größten Hüftenbreite $85 \mathrm{~cm}$, Kopfumfang 55,5 cm. Neurologischer Befund und Befund an den inneren Organen ohne Besonderheiten. Tèmperatur 35,6 ${ }^{\circ}$. Pulsfrequenz 60-70, zeigte respiratorische Schwankungen. Röntgenologischer Befund: Sella normal, Keilbein-Stimbeinhöhle und Arcus superciliaris normal. Persistieren sämtlicher Epiphysenfugen, allerdings ist bei der Jugend des Pat. (163/4 Jahre) nur die proximale Phalange des $\mathrm{Os}$ metacarpale pollicis zu verwerten, die am frühesten, und zwar bis zum 16. Lebensjahre verknöchert. Eine auffallende Hypotonie der Gelenke. Der Tonus der Muskulatur schwach. Die Körperformen weich und rund. Hoden different, der linke bedeutend kleiner als der rechte. Urinuntersuchung: Tagesmenge $750 \mathrm{ccm}$, spez. Gew. 1020, $\mathrm{E}+Z$. negativ.

Morphologische Blutuntersuchang: Rote Blutkörperchen 6300000 Hämoglobin 95\%, weiße Blutkörperchen 5600, davon Neutrophile 43\%, Eosiñophile $10 \%$, Lymphocyten $28 \%$, Mononucleäre und Übergangsformen $14 \%$, Mastzellen 5-6\%. Dies ist das Resultat oft wiederholter Zählungen. Alimentäre Glykosurie bis $225 \mathrm{~g}$ Traubenzucker geprüft: negativ. Nach $15 \mathrm{~g}$ Traubenzucker $1 \mathrm{ccm}$ Suprarenin und 0,005 Cocain keine Glykosurie. Nach 0,001 Phloridzin, subcutan Zucker + .

Die Urintagesmenge stieg nach Suprarenin auf 1300, spez. Gew. 1010. Nach Suprarenin starkes Herzklopfen, Hitzegefühl, Kopfschmerzen, „die Herzschläge donnern", Pulsfrequenz 100, Puls sehr labil. Beklemmungsgefühl, Tremor, Blässe, geringer Schweißausbruch und Durstgefühl. Der hieraus folgenden vermehrten Flüssigkeitsaufnahme ist wohl die stark vermehrte Urinausscheidung zuzuschreiben. Der Blutdruck, der normalerweise 100-105 betrug, stieg nach Suprarenin innerhalb 20 Minuten gleichmäßig auf 110 an und war nach $2 \frac{1}{2}$ Stunden wieder normal. Die Körpertemperatur stieg um 1/2 Grad. Nach Pilocarpin starker Schweißausbruch, keine Salivation. Nach Pituglandol Temperaturanstieg um einige Zehntelgrad. Urintagesmenge unverändert. Einige Tage nach der Aufnahme wurde mit einer vorsichtigen Schilddrüsentherapie begonnen. Es wurden nur kleine Dosen' bis zu dreimal täglich eine Thyreoidintablette 0,1 (Merck) gegeben. Sofort machte sich ein Anstieg der Urintagesmenge von $700 \mathrm{ccm}$ nach und nach bis auf $1400 \mathrm{ccm}$ bemerkbar (Wirkung als Diureticum). Etwas später begann die Körpertemperatur, die sich vorher ständig unter 36 hielt, zu steigen, sie schwankte nun zwischen 36 bis $37^{\circ}$. Urinmenge und Körpertemperatur sanken sofort wieder nach Aussetzen der Schilddrüsentherapie. Auch das Blutbild änderte sich. Nachdem einige Zeit geringe Dosen Thyreoidin gegeben waren, ergab die morphologische Untersuchung folgendes: Rote Blutkörperchen 5600 000, Hämoglobin 95\%, weiße Blutkörperchen 6700, davon Neutrophile 50\%, Eosinophile 19\%, Lymphocyten $20 \%$, Mononucleäre und Übergangsformen $11 \%$, Mastzellen $2 \%$. Nach gesteigerter Schilddrüsentherapie: Rote Blutkörperchen 4500 000, Hämoglobin 90\%, weiße Blutkörperchen 6000, davon Neutrophile $56 \%$, Eosinophile 12\%, Lymphocyten $90 \%$, Mononucleäre und Übergangsformen $11 \%$, Mastzellen $2 \%$. Eigentümlich war das Verhalten des Blutbildes, als bei Schilddrisentherapie $0,75 \mathrm{ccm}$ Suprarenin gegeben wurden. Unmittelbar vor der Injektion wurde das Blut untersucht, und es fanden 
sich rote Blutkörperchen 5100000 , weiße Blutkörperchen 5600 , davon Neutrophile $56 \%$, Eosinophile $12 \%$, Lymphocyten 19\%, Mononucleäre und Übergangsformen $11 \%$, Mastzellen $2 \%$. 2 Stunden nach der Injektion fanden sich rote Blutkörperchen 6400000, weiße 6100, davon Neutrophile 86\%, Eosinophile 5\%, Lymphocyten $4 \%$, Mononucleäre und Übergangsformen 4\%, Mastzellen $1 \%$. Gleichzeitig stieg die Körpertemperatur um $1 / 2$ Grad. Es trat Herzklopfen und Zittern am ganzen Körper ein, und Pat. hatte ein starkes Hitzegefühl. Dagegen hatte Suprarenin bei gleichzeitiger Schilddrüsentherapie eine weitere Steigerung der Urintagesmenge nicht zur Folge. Nach Pituglandol trat bei gleichzeitiger Schilddrüsentherapie ein Temperaturanstieg um $4 / 10^{\circ}$ ein. Eine unangenehme Nebenerscheinung der etwa 3 Wochen fortgesetzten Medikation war eine auftretende Steigerung der Pulsfrequenz und große Labilität des Pulses. Diese Erscheinungen schwanden beim Heruntergehen bis auf 1 Tablette Thyreoidin vollständig. Eine weitere Störung bei höheren Thyreoidingaben waren Klagen über Knochenschmerzen und ziehende Schmerzen in allen Gliedern. Nachdem einige Tage mit der Schilddrüsentherapie ganz ausgesetzt war, worauf Körpertemperatur und Urintagesmenge wieder sanken, wurden Hypophysistabletten 0,1 gegeben, und zwar bis auf 3 Tabletten täglich steigend. Die Körpertemperatur stieg auch nach Hypophysistabletten und hielt sich zwischen $35,8-36,5^{\circ}$, so deutlich und prompt wie beim Thyreoidin war die Steigerung nicht. Auch die Urintagesmenge stieg bis auf $1000 \mathrm{ccm}$, beim Thyreoidin dagegen bis auf 1400. Die störenden Erscheinungen des Pulses traten nach einiger Zeit auch ein, aber nicht so heftig wie beim Thyreoidin. Nach 20 tägiger Behandlung mit Thyreoidin hatte Pat. zum erstenmal eine Erektion. Die Erektion trat unabhängig von irgendwelchen Vorstellungen auf. Sie löste auch keine erotisch gefärbten Vorstellungen aus, war weder von Lustgefühl noch von Schamgefühl begleitet. Pat. empfand die Erektion nur als etwas Fremdes, unangenehm und störend. Die Erektionen wiederholten sich in den nächsten Tagen einige Male, schwanden aber beim Aussetzen und bei kleinen Gaben von Thyreoidin wieder vollständig. Erst sehr viel später, ca. 11/2 Jahr, traten seltene Erektionen auch bei Hypophysismedikation und kleinen Gaben von Thyreoidin auf.

Die Medikation wurde lange Zeit fortgesetzt bei ganz kleinen Dosen und zwischen Schilddrüse und Hypophysis abwechselnd mit eingelegten längeren Pausen. Während der klinischen Beobachtungszeit besserte sich das Allgemeinbefinden nur wenig. Pat. klagte oft über Kopfschmerzen. Nur der Appetit besserte sich, und der Stuhlgang, der vorher angehalten war, wurde regelmaßig.

Die intellektuellen Fähigkeiten des Pat. sind seinem Alter entsprechend. Insbesondere hat er ein gutes Gedächtnis und Beobachtungsgabe, doch beschränkt sich sein Interesse auf die allernächste Umgebung. Beí der Wiedergabe von Erlebnissen bringt er nur eine Aneinanderreihung kleiner Begebenheiten, die er weitschweifig mit unwichtigen Einzelheiten vorbringt, wobei er möglichst seine Person in den Mittelpunkt rückt. Es fehlt seiner Wiedergabe der Zusammenhalt und ein leitender Gedanke. Es interessiert ihn alles nur soweit er es mit seiner Person in Zusammenhang bringen kann. Das Verständnis und die Verwertung des Erlebten sind oberflächlich. Im ganzen macht er einen unreiferen Eindruck, als seinem Alter entspricht. Sein Beschäftigungsdrang ist gering. Seine Spiele sind kindlicher Natur, zu ernstlicher Beschäftigung zeigt er keine Neigung. Bei seinen Spielen zeigt er wenig Phantasie und bevorzugt mechanische Spiele. Pat. ist fromm und besucht gerne die Kirche. Seine Krankheit erscheint ihm sehr wichtig und interessant, er sucht wohl auch durch Klagen und Übertreibung die Aufmerksamkeit auf sich zu lenken. Er fühlt sich leicht zurückgesetzt und bringt über das Essen und das Personal allerlei Beschwerden an. Sexuell ist er ganz unaufgeklärt, entsprechenden Fragen gegenüber ist er völlig ratlos. 
Einige Male klagte Pat. über Übelkeit und Brechreiz. Während der Beobachtungszeit wurden zwei Migräneanfälle und eine eintägige gedrückt-gereizte Verstimmung beobachtet. Einige Tage nach der Entlassung hatte Pat. den Schulbesuch wieder aufgenommen bei fortgesetzter Behandlung. Einen Monat nach der Entlassung schrieb der Vater des Pat., der seinen Sohn ein Vierteljahr lang nicht gesehen hatte „Alfred war munterer, ging elastischer und erträgt Strapazen leichter als früher. Der Stimmwechsel hat eingesetzt". Die eigenen Briefe des Pat. sind dagegen voll Klagen.

Im Februar 1916 machte Pat. einen ca. 8 Tage dauernden migräneartigen $\mathrm{Zu}$ stand durch. Mitte Februar wurde mit der Therapie ausgesetzt und Mitte März wiederholte sich der migränenartige, einige Tage dauernde Zustand.

Mitte April 1916 erneute Untersuchung. Die Adipositas hatte abgenommen, das Gesicht war schmäler geworden; die eunuchoide Fettverteilung war nicht verändert. Die Stimme war im Wechsel.

Erneute Untersuchung $1 \mathrm{Jahr}$ nach Einsetzen der Behandlung und nach längerer Pause der Medikation. Keine Adipositas, eunuchoide Fettverteilung noch deutlich. Die Muskulatur ist fester und zeigt mehr Form. Schamhaare haben erheblich zugenommen, auch in der Achselhöhle setzt Behaarung ein. Stimmwechsel noch nicht beendet. Keine Erektionen. Körpermaße: Körpergröße 165,5 cm (vor $1 \mathrm{Jahr} 162,5 \mathrm{~cm}$ ), Spannweite $172 \mathrm{~cm}$ (vor I Jahr $170 \mathrm{~cm}$ ), Scheitel-Damm 83,5 cm (vor $1 \mathrm{Jahr} 82,5 \mathrm{~cm}$ ). Ob und wie das Wachstum der verschiedenen Körperteile vom normalen abweicht, wäre sehr interessant festzustellen. Leider stehen mir keine Zahlen zur Verfügung. Bekanntlich ist ja das Wachstum der einzelnen Körperteile und die Verhältnisse des Körperbaues in den verschiedenen Lebensaltern ein durchaus verschiedenes.

Pat. zeigte bei der jetzigen Untersuchung keine Überempfindlichkeit gegen Suprarenin.

Blutuntersuchung: Rote Blutkörperchen 6250 000, Hämoglobin 90\%. Weiße Blutkörperchen 4500, davon Neutrophile 66,5\%, Eosinophile 8,5\%. Lymphocyten $20 \%$, Mononucleär- und Übergangszellen 3,5\%, Mastzellen $1,5 \%$.

Urintagesmenge $1500 \mathrm{ccm}$, spez. Gew. 1020, Röntgenologischer Befund unverändert. Migräneanfälle waren in der Zwischenzeit verschiedentlich aufgetreten, auch Kopfschmerzen waren häufig und hinderten ihn in der Schule. Von jetzt ab nahm Pat. nur noch Hypophysistabletten in kleinen Dosen und längeren Zwischenräumen zwischen je 100 Tabletten.

Anfang Februar 1917 verfiel Pat. in einen 20 stündigen Schlafzustand. 5 Tage vor dem Schlafanfall lag Pat. 2 Tage wegen heftiger Kopfschmerz zu Bett. An dem Tage, an dem der Schlafzustand einsetzte, fuihlte Pat. von Mittag an zunehmende Müdigkeit, alles wurde ihm schwer, er konnte sich nicht zum Arbeiten aufraffen. Abends $a ß$ er fast nichts. Um $1 / 28$ Uhr legte er sich nieder und schlief sofort ein. Abends um $10 \mathrm{Uhr}$ versuchte ein Mitschüler vergebens ihn zu wecken. Am anderen Morgen war Pat. durch nichts zu erwecken, es wurde deshalb zum Arzt geschickt. Nach 20 Stunden wachte Pat. mit starker Dösigkeit, Kopfschmerzen und Gliederschmerzen auf. Während des Schlafes keine Zuckungen, kein Urin-, kein Kotabgang. Appetit sehr mäßig, dagegen vermehrtes Durstgefühl. Noch 4 Tage fühlte sich Pat. sehr müde und abgeschlagen und war sehr reizbar. Dann war der Zustand abgeklungen. Pat. hatte in der Zeit vorher angestrengt arbeiten müssen, da er sich auf ein Examen vorbereitete.

Erneute Untersuchung Ende März 1917.

Seit dem Schlafzustand kein weiterer Anfall. Nach angestrengter Schularbeit reizbar. Alle 14 Tage einmal ein Kopfwehtag mit hochgradiger Reizbarkeit. Auf Brom keine Besserung. Stimmwechsel fast vollendet, keine Adipositas, aber noch 
eunuchoide Fettverteilung, besonders deutlich an der Brust. Die Behaarung hat gute Fortschritte gemacht, auch an der Linea alba beginnend. Muskulatur gut entwickelt.

\begin{tabular}{|c|c|c|c|c|}
\hline & rmabe & 1917 & 1916 & 1915 \\
\hline Größe. & . . . & $167 \mathrm{~cm}$ & $165,5 \mathrm{~cm}$ & $162,5 \mathrm{~cm}$ \\
\hline Spannweite & . & 172,5 & 172,0 & 170,0 \\
\hline Scheitel-Damm & 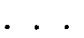 & 85,0, & $83,5 \quad$, & 82,6 \\
\hline
\end{tabular}

Nach Angabe der Mutter ist Pat. selbständiger geworden, zeigt aber sonst in seinem Charakter keine bemerkenswerte Änderung. Erektionen sollen nur selten, und zwar nur in den Zeiten der Medikation aufgetreten sein.

Erneute Untersuchung im März 1919 (20. Lebensjahr).

Pat. hat noch hin und wieder bis Anfang dieses Jahres Hypophysistabletten genommen. Seit Ende 1917 treten Erektionen auf und sind mit der Zeit häufiger geworden; Anfangs noch ohne sexuelle Empfindungen, seit Mitte 1918 mit sexueller Gefühlsbetonung, bald darauf traten auch die ersten Pollutionen ein. Pat. hat eine Neigung zu einem gleichaltrigen jungen Mädchen. Noch kein Geschlechtsverkehr, aber in letzter Zeit starker Geschlechtstrieb, so daß er darunter leidet. Nach Hypophysistabletten bemerkte Pat. eine Zunahme des Geschlechtstriebes. Schlaf- oder Schwindelanfälle sind nicht mehr aufgetreten, die Migräneanfälle sind viel seltener geworden, auch die Kopfschmerzen haben nachgelassen. Er verspürt noch häufig Parästhesien, besonders in der Ruickenhaut. Anfang 1918 wurde Pat. zum Militär eingezogèn und hat den Dienst als Sanitätsmann gut ausgehalten.

Bei der Betrachtung fällt jetzt nur noch eine etwas starke Taillenbildung auf. Fettverteilung und Behaarung sind normal, auch der Bartwuchs an der Oberlippe hat begonnen.

Körpergröße: $170 \mathrm{~cm}$, davon Scheitel-Damm $88 \mathrm{~cm}$. Es zeigt sich also, daß das Längenwachstum ganz erheblich zugunsten der Oberlänge fortgeschritten ist. Wenn wir nochmals diese Maße des Längenwachstums aus den verschiedenen Jahren vergleichend nebeneinander stellen, so ist diese Verschiebung zugunsten der Oberlänge sehr deutlich.

\begin{tabular}{lccc}
\multicolumn{3}{c}{ Körpergröße } & Scheitel-Damm \\
1915 . . . . . . . $162,5 \mathrm{~cm}$ & $82,5 \mathrm{~cm}$ \\
1916 . . . . . . . 165,5, & 83,5, \\
1917 . . . . . . . 167,0, & $85,0 \%$ \\
1919 . . . . . . . 170,0, & $88,0 \%$
\end{tabular}

Pat. machte einen geistig regsameren, lebhafteren Eindruck. Es zeigte sich, daß er alle Vorgänge der letzten Zeit mit Interesse verfolgt hatte. Die Intelligenz hatte sich normal weiterentwickelt. Ende 1917 hatte er das Examen zu Obersekunda gut bestanden.

In Fall 4 finden sich als hereditäre Momente verspätete Pubertätsentwicklung beim Vater und Migräneanfälle bei der Mutter.

Die Beobachtung erstreckt sich auf einen Zeitraum von $3^{1 / 2}$ Jahren. Während dieser Zeit sind die körperlichen Symptome des Eunuchoidismus völlig geschwunden.

Bemerkenswert ist die offenbare Einwirkung der therapeutisch gegebenen Thyreoideatabletten auf das Auftreten der ersten Erektion, auch Hypophysistabletten hatten, aber in weit schwächerem Maße, dieselbe Wirkung. Ein weiterer sicherer Einfluß der Therapie war 
auf die subnormale Körpertemperatur, die abnorm niedrige Urintagesmenge und auf das Blutbild zu konstatieren.

Der Verlauf war kurz folgender: Als erstes Zeichen des Einflusses der Geschlechtsdrüsenfunktion kamen seltene Erektionen, Scham- und Achselhaare. Kurz danach setzte der Stimmwechsel ein. Das Blutbild zeigte sich dem normalen genähert. Dagegen war nach einem Jahre die Fettverteilung noch nicht merklich beeinflußt, und das Längenwachstum hatte sich noch weiter zugunsten der Unterlänge verschoben. Kopfschmerzen und Migräneanfälle zeigten sich nicht beeinflußt, und nach anderthalb Jahren trat ein 20stündiger Schlafzustand auf. Bei der wenige Wochen später erneuten Untersuchung war der Stimmwechsel fast vollendet, die Behaarung der Schamgegend begann den männlichen Geschlechtscharakter anzunehmen. Das Längenwachstum hatte sich ganz auf die Oberlänge beschränkt. Die Fettverteilung war noch deutlich eunuchoid. Bei der Untersuchung zwei Jahre später sind Fettverteilung und Behaarung normal, letztere sogar stark ausgebildet. Die weiteren $3 \mathrm{~cm}$ Längenwachstums hatten sich ganz auf die Oberlänge beschränkt. Der Brustkorb war in den Schultern bedeutend breiter geworden. Die Stimme ist tief. Gesichtsausdruck und Auftreten sind männlich. Patient ist geistig regsamer geworden. Nach Angabe der Eltern hatte auch die psychische Pubertätsentwicklung mit ihren bekannten Erscheinungen, Flegeljahre, Drang nach Selbständigkeit, Neigung zu Ưberhebung, erste Liebe usw. mit der physischen gleichen Schritt gehalten. Die Kopfschmerzen haben sich gebessert, die Migräneanfälle sind seltener und leichter geworden.

Als Kind und in den Schuljahren war Patient still, artig, gern für sich allein, ängstlich, weichlich, launenhaft, empfindlich, körperlich träge, schwerfällig. Er zeigte mangelnde Aufnahmefähigkeit und Verwertung gefühlsbetonter Erlebnisse, Mangel an Phantasie beim Spiel. Er war indifferent und ohne Ehrgeiz, zeigte kein Bedürfnis nach Anschluß und Freundschaft. Er war leicht gereizt mit Neigung zum Explodieren, litt an Kopfschmerzen, Migräneanfällen und bekam einen 20 stündigen Schlafanfall.

Es handelt sich um einen völlig ausgeheilten Fall von temporärem Eunuchoidismus. Einen solch glatten Verlauf sieht man in der Regel nicht. Vielmehr ist es auch nach Eintritt der Geschlechtsreife meist. leicht, aus noch vorhandenen körperlichen Restsymptomen die Diagnose des durchgemachten Utbergangseunuchoidismus zu stellen. Ich bin deshalb der Meinung, daß in diesem Falle doch der eingeleiteten Therapie einige Wirkung einzuräumen ist.

Ich will hier noch erwähnen, daß es mir wiederholt gelang, Migräne bei Anzeichen von Adipositas durch Hypophysistabletten auffallend günstig zu beeinflussen. 
Fall 5. 25 Jahre alt, Schlächter. Der Vater in den letzten Lebensjahren geisteskrank, starb in einer Anstalt (Paralyse?). Die Mutter leidet an Rheumatismus. Zwei seiner Brüder in den ersten Lebensjahren an Krämpfen gestorben. Zwei lebende gesunde Schwestern. Bis zum sechsten Jahre Bettnässer. Als Kind Masern, Diphtherie, Lungenentzündung. War still, ängstlich, für sich allein, das Lernen in der Schule machte ihm wenig Freude, hatte Furcht vor den Lehrern, war empfindlich und hatte keine Freunde. Im 13. bis 14. Lebensjahre Nachtwandeln. In späteren Jahren oft Verstimmungen ohne Anlaß und Kopfschmerzen. War als Kind eher klein, Ende des 18. Lebensjahres $160 \mathrm{~cm}$ groß. Danach stark und plötzlich gewachsen und magerer geworden, etwa bis zum 23. Jahre. Mit 20 Jahren ist der früher kleine Geschlechtsteil gewachsen und die ersten Scham- und Achselhaare aufgetreten. Etwas Bartwuchs seit dem 23. Jahre. Erste Erektion im 20. Lebensjahre, Erektionen sind selten, Pollutionen gar nicht vorhanden. Keine Libido, auch bei den Erektionen angeblich keine erotischen Emptindungen. Geht nicht auf den Tanzboden, trinkt keinen Alkohol, wenig Kameradschaft, oft mißgestimmt, unzufrieden, müde und unlustig zur Arbeit. Gehört einem religiösen Verein und dem Jünglingsverein an. Fühlt sich leicht gekränkt, wird dann gereizt und jähzornig. Von jeher viel an Kopfschmerzen gelitten und seit $1 \frac{1}{2}$ Jahren an kurzen Schwindelanfällen. Nach einem Bericht waren bei ihm schon vor $1 / 4 \mathrm{Jahr}$,Anfälle von ohnmachtsartiger Schwäche“" ärztlich beobachtet worden.

Graziler Knochenbau. Die eunuchoide Disproportion des Skeletts ist deutlich. Körpergröße $173 \mathrm{~cm}$ bei einem Körpergewicht von $63,5 \mathrm{~kg}$. Spannweite $184 \mathrm{~cm}$. Die Unterlänge überragt die Oberlänge, die Armlänge, $76 \mathrm{~cm}$, ist in der Körpergröße statt 2,5 2,3 mal enthalten. Kopfumfang $55 \mathrm{~cm}$. Breite Jochbeine, Prominentia laryngea nur beim Schlucken sichtbar, beiderseitiger Plattfuß, das Fettpolster ist schlecht entwickelt, am besten an der Brust und am Mons pubis. Kopfhaar dicht und stark, Bartwuchs spärlich an der Oberlippe, Achselbehaarung spärlich, Schambehaarung mäßig, sich nicht auf die Linea alba fortsetzend und

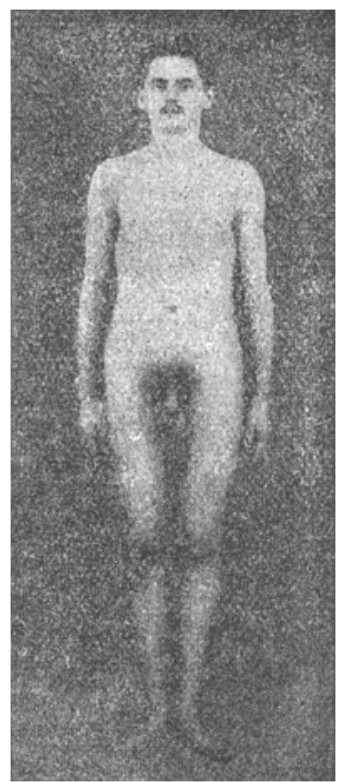

Abb. 5 oben in gerader Linie abschneidend. Linea alba nicht pigmentiert. Die Haut ist zart und weiß. Die Muskulatur ist schwach entwickelt. Röntgenologischer Befund: Die Sella ist eher groß. Epiphysenlinien am distalen Ende vom Radius offen, von der Ulna teilweise offen, teilweise geschlossen, in den verknöcherten Teilen Compactainseln. An den Phalangen finden sich strichförmige Compactainseln an Stelle der Epiphysenlinien.

Urintagesmenge bei $3500-4000 \mathrm{ccm}$.

Alimentäre Glykosurie negativ.

Körpertemperatur normal.

Innere Organe und neurologischer Befund o. B.

Die Stimmlage ist hoch.

Die Intelligenz entspricht dem Durchschnitt. Pat. lag meist beschäftigungslos im Bett, ohne die Umgebung zu beachten. Er zeigte eine auffällige Bewegungsarmut. Gegen die Kameraden auf der Station war Pat. verschlossen, abweisend und reizbar. Die Stimmung war gedrückt, ängstlich, verbittert. Er klagte über 
Kopfschmerzen, Zittern, Gelenkschmerzen, Schlaflosigkeit, Appetitlosigkeit, Schwächeanfälle und Brechreiz. Ich beobachtete einen epileptiformen Anfall, Pat. streckte sich plötzlich, "dann traten klonische Zuckungen und Röcheln auf, und danach tiefer Schlaf. Er selbst weiß nichts von einem Anfall. Am Tage darauf verstimmt, mißmutig, gereizt, verlangsamt und schwerfällig. Außerdem beobachtete ich einen kollapsartigen Zustand, Pat. wurde auffallend blaß, dann bewußtlos, der Puls war klein und langsam, der Körper mit kaltem Schweiß bedeckt, danach tiefer Schlaf. Ferner wurden einige Verstimmungen beobachtet.

Fall 5 ist ein Fall von stark verzögertem und verlangsamtem Eintritt der Geschlechtsreife, bei dem jetzt im 25. Lebensjahre erst Spuren einer Geschlechtsdrüsenfunktion vorhanden sind. Bis zum 6. Jahre Bettnässen. Als Kind still, ängstlich für sich allein, später träge, schwer zugänglich, stumpf, indifferent, verbittert, leicht

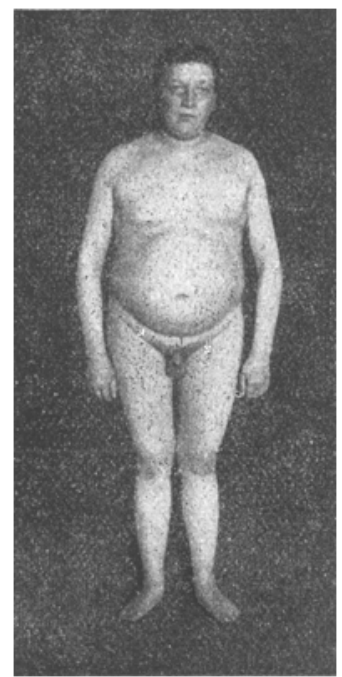

Abb. 6 . gekränkt, reizbar, jähzornig, sehr fromm.

Leidet an Kopfschmerzen, Schwindelanfällen, Verstimmungen mit gesteigerter Reizbarkeit. Außer diesen wurde ein epileptiformer Anfall und ein kollapsartiger Zustand beobachtet.

Fall 6. 37 Jahre alt, Schneider. Der Vater war nervös. Vier lebende verheiratete Schwestern, die Kinder haben. Pat. war das vorletzte Kind, das letzte Kind starb bald nach der Geburt. Zur rechten Zeit Sprechen und Laufen. Mit 7 Jahren Typhus. In der Kindheit still und schüchtern, wenig Kameraden, in der Schule gut gelernt, seit dem 17. Lebensjahre nicht mehr gewachsen. Mit 15 Jahren einige Erektionen, die dann ganz wieder bis zum 22. Jahre aufhörten. In dieser Zeit Zunahme des Fettpolsters und viel Kopfschmerzen. Mit 23 Jahren erster Geschlechtsverkehr, mit 24 Jahren Ehe. Pat. wurde lebhafter und gründete ein eigenes Geschäft. Pat. hat vier Kinder, von denen das letzte kurz nach der Geburt starb. Seit 5 Jahren zunehmende Fettentwicklung und Nachlassen der Libido. Seit einem Jahr keine Libido und kein

Geschlechtsverkehr mehr. Vom 27. bis 34. Jahre hat er reichlich alkoholische Getränke getrunken.

Die Körperbehaarung soll nie erheblich stärker gewesen sein, auch die Stimme ist immer hoch gewesen.

Graziles Skelett. Die eunuchoide Disproportion wird durch die Körpermaße noch deutlich. Körpergröße $162 \mathrm{~cm}$, Spannweite $172 \mathrm{~cm}$. Armlänge $69 \mathrm{~cm}$, geht anstatt 2,5 nur 2,3 mal in der Körperlänge auf. Der Schädel ist länglich, das Hinterhaupt steil, Unterkiefer massig, Prominentia laryngea auch beim Schlueken nicht sichtbar, Genu-valgum-Stellung der Beine, beiderseitiger Plattfuß. Es finden sich also genügend für das eunuchoide Skelett charakteristische Merkmale. Erheblich sind die Fettansammlungen an der Brust, Hüften, Außenseite der Oberschenkel, Unterbauchgegend und dem von dieser durch eine tiefe Furche abgesetzten und vorspringenden Mons pubis. Das Kopfhaar ist gut entwickelt. An Stelle des Bartes Lanugobehaarung. Schambehaarung nur an der Peniswurzel. Die Haut ist zart und pigmentarm, die Körperformen weich und rund. Muskulatur mittelkräftig. Äußere Genitale klein. 
Röntgenologischer Befund: Sella normal, Unterkiefer massig, die Epiphysenlinien wurden nur am distalen Ende von Radius und Ulna und an der Hand nachgesehen. Sie waren verknöchert, aber an ihrer Stelle fanden sich Compactainseln und zwar meist strichförmig.

Urintagesmenge 1800-2000 ccm. Alimentäre Glykosurie negativ. Blutuntersuchung: Rote Blutkörperchen 5000 000, Hämoglobin 70\%, weiße Blutkörperchen 4100 .

Die Körpertemperatur war meist unter $36^{\circ}$, die tiefste $35,2^{\circ}$. Sie stieg nach $1 \mathrm{ccm}$ Adrenalin um $8 / 10^{\circ}$. Nach einigen Tagen Thyreoidinmedikation stieg die Temperatur auf $36,8^{\circ}$, nach Aussetzen des Thyreoidins fiel die Temperatur wieder bis $35,3^{\circ}$. Nach Adrenalin lebhafte Überempfindlichkeitsreaktion.

Eunuchoide Stimme. Die Gesichtszuge sind weich und schlaff, das Gesicht hat nichts Männliches. Haltung und Bewegung schwerfällig. und unelastisch. Normale Intelligenz.

Pat. war in der Zeit der erlangten Geschlechtsreife regsam und fleißig, hatte ein kleines Geschäft gegründet und ein kleines Vermögen erworben. Etwa zu der Zeit, wo die Involution der Geschlechtsdrüse einsetzte, ließen seine Regsamkeit. und Fleiß nach, er gab sein Geschäft auf und wollte von seinen Zinsen leben. Er war ein empfindlicher und reizbarer Mensch, litt an Kopfschmerzanfällen, Gelenkschmerzen und Reißen.

Es handelt sich um einen Fall von temporärem Eunuchoidismus in der Pubertät, bei welchem nach vorübergehend erlangter Geschlechtsreife von ca. 10 Jahren schon auffallend früh, etwa im 33. Lebensjahre, die Involution wieder einsetzt. Ohne genaue Anamnese würde man verleitet sein, den Fall zum Späteunuchoidismus zu rechnen. Aber deutlich vorhandene eunuchoide Skelettmerkmale, die nur vor Abschluß der Wachstumsperiode eintreten können, sowie die sichere Vorgeschichte reihen den Fall einer anderen Gruppe ein, die ich in der Einleitung erwähnt habe. Ich habe noch einige andere derartige Fälle gesehen, so z. B. war auch der Vater von Fall 4 ein solcher. Utberhaupt scheint mir die frühe Involution bei später erlangter und verlangsamter Geschlechtsreife das häufigere zu sein. Gar nicht so selten, wie auch im vorliegenden Falle und Fall 3, hört man von den Patienten, daß schon einmal etwa zu der Zeit, in der normalerweise die Pubertät beginnt (14. bis 15. Lebensjahr), einige Erektionen aufgetreten sein sollen, die dann ganz wieder schwanden.

Als Kind war Patient still und schüchtern, gern für sich allein. Mit dem 15. Lebensjahr viel Kopfschmerzen und Zunahme des Fettpolsters, mit 22 Jahren Beginn der Geschlechtsreife. Ging eine Ehe ein, wurde lebhafter, betriebsam und fleißig, gründete ein eigenes Geschäft. Mit ca. 33 Jahren Beginn der Involution, Nachlassen der Libido, Zunahme des Fettpolsters und Abnahme der Körperbehaarung. Gleichzeitig wird Patient interesseloser, untätig, träge, gibt sein Geschäft auf, will von seinem Gelde leben. Dabei empfindsam und reizbar; leidet an Migräneanfällen.

Aus dem, was die Beschreibung der Fälle bietet, möchte ich noch einiges als besonders erwähnenswert hervorheben. 
Alle Fälle, in denen daraufhin untersucht wurde, zeigten eine ausgesprochene Adrenalinüberempfindlichkeit. Diese erscheint mir besonders wichtig für die Genese der vasomotorischen Symptome. Diese Adrenalinüberempfindlichkeit haben die Eunuchoiden nach meinen Erfahrungen mit der größten Zahl der Epileptiker gemeinsam.

Als selteneres Symptom sah ich zweimal das wulstartige Vorspringen der Raphe des harten Gaumens. Parästhesien der Haut (besonders Hautjucken) scheinen nicht ganz selten zu sein.

Utber leichte neuritische und rheumatische Beschwerden wird in einigen Fällen geklagt.

Allen gemeinsam ist ein flaches Fußgewölbe, meist besteht echter Plattfuß.

Nicht selten hört man von den Patienten, daß zu der Zeit, in der etwa normalerweise die Pubertätsentwicklung einsetzt (14. bis 15. Lebensjahr), vereinzelte Erektionen aufgetreten seien, die dann ganz geschwunden sind, oder bei Besserung (Utbergangseunuchoidismus) erst nach Jahren wieder auftraten. Utber die mehrfach erwähnten Compactainseln an den Epiphysenlinien möchte ich folgendes sagen. Schon vor einigen Jahren hatte ich $^{1}$ ) bei der Beschreibung solcher Compactainseln im Röntgenbilde die Ansicht vertreten, daß es sich um versprengte und später verknöcherte Teile der Epiphysenscheibe handele. Ich bin seither zu der Utberzeugung gekommen, daß die Compactainseln ein Symptom einer Störung im Epiphysenschluß darstellen, und zwar einer verlangsamten und verzögerten und infolge davon unregelmäßig erfolgenden Verknöcherung.

Man kann die Compactainseln mit gutem Recht bei der Diagnose des Eunuchoidismus mit verwenden, jedoch nur insoweit als sie zu den Erscheinungen des Hypogenitalismus gehören. Sie sind also nur für einen solchen beweisend, nicht aber für einen überstandenen Eunuchoidismus. Wenn alle übrigen Skelettsymptome des Eunuchoidismus fehlen, ist hiermit allein nichts zu beweisen. Nach meinen Beobachtungen geht die Intensität der Störung im Epiphysenschluß dem Grad des Geschlechtsdrüsenausfalles parallel. Die Compactainseln sind die leichteren Grade resp. auch die Reste der Störung im Epiphysenschluß, denn daß auch diese sich ganz verlieren könnten, ist wohl unwahrscheinlich. Wir haben in ihnen also ein sicheres Merkmal, um auch später noch einen überstandenen Hypogenitalismus zu erkennen.

Eine Abhandlung über psychische Veränderungen beim Eunuchoidismus sollte zweckmäßig mit einem Studium bei den Kastraten beginnen, die zur Zeit noch in der Skopzensekte genügend Vertreter haben. Einem solchen Vorhaben stellen sich aber besonders jetzt

1) H. Fischer, Beitrag zur Kenntnis der Skelettvarietäten. Fortschritte auf dem Gebiet der Röntgenstrahlen. Bd. XIX. 
erhebliche äußere Schwierigkeiten entgegen. Was ich während des Krieges in Rußland und auf dem Balkan über die Skopzen erfahren konnte, war nichts Positives. Ein serbischer Arzt erzählte mir, daß bei ihren Versammlungen religiöse Verzückungszustände eine Rolle spielen sollen. Es sei auch darauf hingewiesen, daß man beim Studium des Eunuchoidismus meiner Ansicht nach nicht, wie das oft in der Literatur geschehen ist, Kastraten, Klimakterische und Eunuchoide als etwas Gleichwertiges hinstellen kann. Nach ihren ätiologischen Entstehungsbestimmungen sind das Experiment der Kastration, die physiologische Involution und die Konstitutionsanomalie des Eunuchoidismus etwas durchaus Verschiedenes.

Von der Wirkung der Kastration auf Tiere wissen wir, daß diese außer dem Geschlechtstrieb an Lebhaftigkeit, Bewegungsdrang, Feuer und Aggressivität verlieren, dagegen nicht untauglicher zu körperlichen Arbeiten werden. Im Gegenteil, sie werden durch die oben angeführten Ausfallserscheinungen leichter lenkbar, und so sind manche Tiere wegen ihrer Brauchbarkeit als Arbeitstiere nach der Kastration besonders geschätzt. Von kastrierten Menschen sagt man, sie seien phlegmatisch, faul, geizig, geldgierig, egoistisch, grausam, schlau, melancholisah, niemals aber finden sich Belege für eine Verminderung der intellektuellen Kräfte oder gar für die Umänderung in den anders geschlechtlichen Typus. Die Kastraten gelten als ungeeignet zu produktivem Schaffen und organisatorischer Tätigkeit. Doch erscheint wahrscheinlich, daß Eunuchen an gewissen Höfen eine einflußreiche Stellung als Hofbeamte eingenommen haben. Auch von Virtuosentum wird berichtet. Ihre Erfolge als Sänger haben sie der Eigentümlichkeit der Kastratenstimme zu verdanken, die besonders in Italien bei religiösen Gesängen geschätzt wurde. Virtuosentum bei Anlagen für gewisse Künste, insbesondere für Musik, findet sich aber auch nicht selten schon bei Kindern. Bei solchen Kindern sehen wir einen deutlichen Kontrast zwischen den Leistungen des Virtuosentums und der sonstigen durchaus kindlichen Psyche. Höherwertige künstlerische, vor allem produktive Leistungen, liefert erst die reif gewordene Persönlichkeit, wenn die Leitung der künstlerischen Begabung von dieser übernommen ist und in der Kunst ihren Ausdruck sucht. Als Ausnahme finden sich hier nur wenige Genies, die dann aber auch als Kind schon in anderen Gefühlswerten voraus waren und bei welchen sich nicht selten schon früh die ersten Spuren eines Liebeslebens bemerkbar machten.

Die bisherigen Angaben über das Leben und die Taten großer Eunuchen aus der Geschichte sind natürlich schwer zu verwenden. Es fehlen, da ja die wissenschaftliche Erforschung der physiologischen Folgeerscheinungen der Kastration noch eine junge ist, in den vor- 
handenen Aufzeichnungen alle Anhaltspunkte für den Zeitpunkt und die Vollständigkeit der vorgenommenen Kastration.

Hiervon machen auch die Beispiele, die Rieger ${ }^{1}$ ) anführt, keine Ausnahme. Nach Riegers Angabe verfolgt seine Arbeit den Zweck, den Mythus von der Wichtigkeit der Testikel für Intelligenz und Energie zu zerstören und die Emanzipation der Psychiatrie vom medizinischen Aberglauben zu fördern. Das, was über die Wirkung der Kastration auf die sekundären Geschlechtsmerkmale bekannt war, rechnet Rieger zum Teil ,ins Reich der Mythologie“ oder in „das gefährliche Gebiet der Jagdgeschichten". Rieger beobachtete einen Fuhrknecht, dem mit 21 Jahren die Testikel völlig zerquetscht wurden. Ob dieser Fall einer völligen Kastration gleichzusetzen ist, erscheint mir fraglich, es können wohl Teile von dem widerstandsfähigeren innersekretorischen Gewebe erhalten geblieben sein. Rieger hielt auf Grund dieser Beobachtung ein für allemal den Punkt für erledigt, daß die Morphologie der Erwachsenen durch die Kastration nicht berührt wird. Ebenso kann er bestimmt die Frage verneinen, daß die Entfernung der Testikel dem allgemeinen Körperzustand durch Verlust der inneren Sekretion der Testikel schadet. In dem von ihm beobachteten Falle bestand nur ein Verlust des Geschlechtstriebes, und der Bartwuchs war sehr schwach. Über das psychische Verhalten wird gesagt, ,der Mann ist ein schuchterner Bauernknecht und zu Äußerungen über seine inneren Zustände wenig veranlagt“. Zur Beantwortung der Frage ,wie die Kastration auf den allgemeinen Körper- und Geisteszustand von sonst normalen Menschen wirkt" scheinen ihm die Skopzen ,deren ganzes Milieu uns verrúckt erscheinen muß" nicht brauchbar. Brauchbar erscheinen ihm einige Männer aus der Geschichte, und zwar der byzantinische Feldherr Narses, der Philosoph Pierre Abilard und der Kirchenvater Origenes. Das Studium dieser drei Manner beweist $\mathrm{ihm}$, daß der Mensch an seinem höchsten geistigen Dasein in Kirche, Staat und Wissenschaft etwas besitzt, was zwar durch den vorhandenen Geschlechtstrieb oft getrübt und gestört werden, aber nicht zerstört werden kann durch den beseitigten Geschlechtstrieb. Durch das Beispiel des Narses speziell findet Rieger mit Sicherheit bewiesen, ,daß die Testikel kein notwendiges Erfordernis fur einen Helden sind". Ich halte diese historischen Personlichkeiten fur ungeeignet zu einer Beweisführung, da bei ihnen natülich zuerst der Beweis zu fuhren wäre, daß es sich wirklich um Kastraten gehandelt hat. Narses soll einen kleinen schwächlichen Körper gehabt haben, demnach durfte er kaum ein Fruhkastrat sein. Er kam erst als Kriegsgefangener in den Palast des Kaisers Justinian I. und wurde so nach und nach Aufseher uber die Archive, Oberkämmerer und Gunstling des Kaisers. Da nun der Name Eunuchos am oströmischen Hofe auch zur Bezeichnung eines Hofamtes, gleichbedeutend etwa mit Kammerherr und Gunstling des Kaisers vorkommt, konnte Narses auch auf diese Weise zu dem Namen Eunuchos gekommen sein. Pierre Abilard soll im 36. Lebensjahr aus Rache kastriert sein. Hier handelt es sich also vorausgesetzt, daß es sich nicht nur um eine mehr oder weniger eingreifende Verstümmelung handelte, nur um einen Spätkastraten. Origines endlich soll sich nur mit Wahrscheinlichkeit als Jingling selbst kastriert haben.

Auf Grund neuerer Forschungen haben wir ohne Frage zur Zeit von einer Einwirkung der Geschlechtsdrüse resp. deren Ausfall auf die Psyche einige Kenntnis. Uber die Folgezustände der Kastration auf die Psyche der geschlechtsreifen Frau liegen reichliche Beobachtungen vor. Die auftretenden nervösen Beschwerden sind im ganzen den

1) Rieger, „Die Kastration“. Jena 1900. 
klimakterischen Ausfallserscheinungen ähnlich, aber heftiger als diese. Es fehlt in der Regel nach der Kastration die im Klimakterium anfänglich noch auftretende zyklische menstruelle Welle, oder die Wiederkehr der Molimina menstrualia sine menstruatione. Biedli) berichtet allerdings auch von einigen Beobachtungen, bei denen diese letzteren Erscheinungen wie im Klimakterium bestanden. Es erscheint mir wahrscheinlich, daß bezüglich des Auftretens dieser Erscheinungen das Alter der Kastrierten eine große Rolle spielt, insofern als die Folgeerscheinungen der Kastration den klimakterischen um so ähnlicher werden, je näher die Kastrierten dem klimakterischen Alter kommen, da die Kastration dann einen mehr auf den Geschlechtsdrüsenausfall eingestellten Organismus vorfindet. Ich halte es aber für nicht nützlich, die Kastration ein künstliches Klimakterium zu nennen, hiergegen sprechen auch die Unterschiede in den somatischen Erscheinungen und andererseits ist' das Klimakterium sicherlich mehr als nur eine Involution der Geschlechtsdrüse. Letztere ist nur das Auffallendste an ihr, weil wir für den Geschlechtsdrüsenausfall die auffallendsten Kriterien haben. Im Vordergrund der Beschwerden kastrierter Frauen stehen vasomotorische Erscheinungen, Wallungen, heiße Utbergießungen, Schweißausbrüche, Ohrensausen, Flimmern vor den Augen, Herzklopfen. Pfister ${ }^{2}$ ) schreibt, auffallend häufig klagen die Operierten über Kopfschmerzen; dabei ist mir aufgefallen, daß diese Schmerzen vielfach das Bild einer Migräne bieten. Ferner wird berichtet von nervösem Erbrechen, Brechreiz, Neuralgien einzelner Nerven, namentlich des Plexus lumbalis, Krampfhusten, chronischer Heiserkeit. Weiter sagt Pfister: „12 Frauen sagen aus, daß sie seit der Operation entschieden reizbarer geworden seien." Ähnliche• Angaben finden sich auch bei ärztlich beobachteten männlichen Kastraten. So schreibt Hirschfeld ${ }^{3}$ ) von einem 49 jährigen Kaufmann, der vor 3 Jahren auf seinen Wunsch wegen perverser sexueller Neigungen kastriert war, ,er zeigt eine erhebliche Affekterregbarkeit". „Er ist sehr gutmütig, gefällig, arbeitsam, lebt jetzt sehr zurückgezogen und geht täglich in die Kirche. Gedächtnis und Intelligenz sind gut und haben gegen früher keine Abnahme erfahren." Von einem anderen mit 21 Jahren kastrierten jungen Manne sagt Hirschfeld: ,Ganz regelmäßig treten Zeiten ein, in denen er sich unwohl fühlt; er leidet dann an Schwindel, Mattigkeit, Kopfweh, starker Gereiztheit und seelischer Verstimmung." Psychische und nervöse Störungen als Begleitsymptome der Involution finden sich auch beim Manne. Die Involution setzt beim Mann durchschnittlich später ein als bei der Frau. Daß der Zeitpunkt der Involu-

1) Biedl, „Innere Sekretion“. 1916.

2) Pfister, Archiv f. Gynäkol. 56, 583. 1898.

3) Hirschfeld, Neurol. Centralbl. 1916. 
tion beim Mann weniger deutlich ist als bei der Frau, liegt wohl lediglich daran, daß ein so sicheres Kriterium wie die klimakterischen Menstruationsanomalien fehlt. Daher wird andererseits wohl wieder die Ätiologie der Involutionsbeschwerden nicht immer erkannt. Der Einfluß der Involution auf das Symptombild bestehender Psychosen wie für das Auftreten frischer Psychosen ist genügend bekannt. Wir kennen nicht nur in der Pubertät, sondern auch im Klimakterium eine gefahrvolle Zeit für die Psyche beider Geschlechter.

Diesen aus dem Negativen zu folgernden Leistungen der Geschlechtsdrüse sind auch positive Leistungen auf psychischem Gebiete einzureihen. Früher schrỉeb man der Geschlechtsdrüse einen wesentlichen Anteil an der gewaltigen Reaktion im psychischen Geschehen zur Pubertätszeit zu. Hellmonth sagt: ,Propter solum uterum mulier est, quod est", und Virchow: „Das Weib ist eben Weib durch seine Generationsdrüse. Alle Eigentümlichkeiten seines Körpers und Geistes, kurz alles, was wir an dem wahren Weibe Weibliches bewundern und verehren, ist eine Dependance des Eierstockes."

Dem muß aber folgendes entgegengehalten werden. Sicher ist, daß die somatische Reife mit Ausbildung aller sexuellen Merkmale ohne entsprechende psychische Reife unter pathologischen Bedingungen vorkommt. Bei der prämaturen Entwicklung auf Grund von Genitaltumoren hält die psychische mit der physischen Reifung nicht gleichen Schritt. Solche Riesenkinder mit prämaturer sexueller Reife zeigen sich vielmehr in ihrem Intellekt- und Gefühlsleben normalen Kindern gleicher Altersstufen gleichwertig. Ferner sind Fälle gesteigerter, nur physischer Entwicklung bei Nebennierentumoren beschrieben.

Dagegen zeigen Fälle mit prämaturer Entwicklung auf Grund von Zirbeldrüsentumoren (und zwar ist in diesen Fällen eine Insuffizienz dieser Drüse anzunehmen) neben der körperlichen auch prämature psychische Entwicklung.

Normalerweise treten nach Marburgs ${ }^{1}$ ) Untersuchungen anscheinend die ersten Involutionserscheinungen an der Zirbeldrüse schon vor der Pubertät ein und leiten so diese gewissermaßen ein. So haben wir wohl Anlaß zu der Annahme, daß erst die Involution dieser Drüse die Reifung des ganzen Organismus einschließlich der Reifung der Geschlechtsdrüse möglich macht, und somit die Folge dieser Reifung der Geschlechtsdrüse, die sexuelle Reife, nur ein Teil wichtiger Entwicklungsvorgänge im Körper und nicht die Ursache dieser ist. Nur so läßt sich eine Unabhängigkeit einzelner Reifungsvorgänge voneinander ungezwungen erklären. Bei dieser Vorstellung haben wir zwei Bedingungskomplexe, die zur Zeit der Reifung des Organismus fehler-

1) Marburg, Arbeiten aus dem neurolog. Institut an der Wiener Universität 1909. 
haft reagieren können. Einmal kann die Involution der Zirbeldrüze eine unvollständige sein, so daß Reize, auf die bestimmte Reifungsvorgänge im Organismus eingestellt sind, ausfallen, andererseits kann das zu reagierende Organ auf vorhandene elektive Reize nicht reaktionsfähig, d. h. krank sein.

Deutliche Geschlechtsdifferenzen zeigen sich schon im Kindesalter, somatisch in Körperform, Fettansatz, Muskulaturentwicklung, Haarwuchs. Beckenunterschiede bestehen nach Fehlings Angaben sogar schon beim Foetus. Psychische Differenzen machen sich im Ausdruck der Bewegung, der Mimik, in Neigungen und Spielen bemerkbar. Diese psychische Differenz der Geschlechtscharaktere im Kindesalter hat natürlich nichts mit der Vorstellung von psycho-sexuellen Empfindungen und Trieben beim Kinde zu tun. Weder der unter pathologischen Bedingungen schon frühzeitig auftretende Detumeszenztrieb beim Kinde, noch alle vor der Pubertät auftretenden Erektionen setzen etwa psycho-sexuelle Empfindungen voraus. Diese Tatsache bestätigen auch die Erfahrungen beim Eunuchoidismus. Es wurde mir wiederholt von Patienten angegeben, daß auftretende Erektionen ohne jede Lustbetonung, im Gegenteil unangenehm empfunden wurden. Ein Liebesleben bei Kindern, wie es von einzelnen Genies bekannt ist, gehört zu seltenen Ausnahmen. Altruistische Gefühle dagegen sind beim Kinde nicht selten, und zwar bei solchen Kindern, bei denen sich nicht der geringste Anhaltspunkt für eine frühzeitig erwachte Sexualität mit erotischen Neigungen findet. Es ist nicht richtig alle höheren Gefühlswerte nur von psycho-sexuellen Empfindungen abhängig zu machen. Zweifelsohne fallen bei normaler Entwicklung das Erwachen psycho-sexuellen Empfindens und Auftreten höherer Gefühlswerte meistens zusammen und erhalten durch das sexuelle Triebleben und seine Folgen ihre bestimmte Färbung und Verwertung, insbesondere die sozialen Gefühle. Wị wissen ferner, daß erotisches Erleben oft mit einer erheblichen Steigerung der Arbeits- und Produktionsenergie einhergehen kann. Die Pathologie lehrt uns aber, daß die prämature psychische Entwicklung nicht von einer gleichwertigen physischen Entwicklung begleitet oder gefolgt $\mathrm{zu}$ werden braucht, es besteht vielmehr eine offensichtliche Unabhängigkeit beider voneinander.

Die Möglichkeit eines Eunuchoidismus im Kindesalter ist gegeben. Sein Erkennen natürlich ist ungleich schwieriger und ich glaube nicht, daß man vor Ende der zweiten Kindheitsperiode, wenn die Differenzierung des Fettpolsters der Geschlechter anfängt, den Eunuchoidismus mit Sicherheit erkennen kann. Hierfür sprechen übrigens auch die anatomischen Befunde am Hoden. Bouin und Ancel ${ }^{1}$ ) fanden folgenden Entwicklungsgang der innersekretorischen Zwischensubstanz

1) Zit. nach Biedl. 
des Hodens: Das erste Erscheinen der Le ydigschen Zellen beim Embryo geht der sexuellen Differenzierung der primordialen Keimzellen voraus. Aus dem strukturellen Verhalten der Zellen schließen die Autoren auf ihre besondere Aktivität im Embryonalleben. Diese Zellen sollen daher beim Embryo durch ihr spezifisches Sekret das Geschlecht der generativen Zellen der Keimanlage im masculinen Sinne bestimmen. Aus dieser besonderen Aktivität erklärt sich wohl auch die Differenzierung des ganzen sexuellen Hilfsapparates beim Embryo. Vom sechsten Monat aber tritt eine Verminderung der Zellen ein, und bei Neugeborenen und Kindern sind keine eigentlichen Zwischenzellen vorhanden. Diese erscheinen erst wieder Ende der zweiten Kindheitsperiode, wenn die Pubertätsentwicklung einsetzt.

Bei zwei im Pubertätsalter stehenden Eunuchoiden konnte ich Erfahrungen sammeln, die zur Aufklärung der Frage beitragen. Von den gut beobachtenden intelligenten Eltern wurde mir in dem einen Fall angegeben, daß ihnen am körperlichen Wachstum des Kindes vor dem 10. Lebensjahre nichts aufgefallen sei. Seit dem 10. Lebensjahre zeige es ,etwas mädchenhafte Formen“. Mit diesem Alter sollen auch die Kopfschmerzen eingesetzt haben. In dem anderen Falle wurde der Beginn mit demselben Symptome auf das 11. Lebensjahr verlegt.

Beide sollen schon in der ersten Kindheit ängstlich und schüchtern gewesen sein, sie spielten lieber für sich allein als mit anderen Kindern. Derartige Angaben finden sich zwar oft in der Vorgeschichte Eunuchoider, aber auch in der Vorgeschichte anderer Krankheiten, insbesondere der Dementia praecox. Auf diese Angaben also kann wohl niemand die Diagnose des Eunuchoidismus stützen wollen.

In der zweiten Kindheitsperiode schlossen sich die beiden Kranken ungern an gleichaltrige Knaben an. Diese waren ihnen zu wüst. Sie waren auch für Eltern und Geschwister schwerer zugänglich als andere Kinder. Hin und wieder waren die Patienten gereizt, launenhaft, empfindlich und resistent. Beim Spiel zeigten sie wenig Selbständigkeit und Phantasie. Ich hatte Gelegenheit, beide Fälle längere Zeit zu beobachten, den einen (Fall 4) mit Unterbrechungen $3^{1 / 2} \mathrm{Jahr}$ lang. Beide kamen in meine Beobachtung erst mit dem 15. resp. 16. Lebensjahre. Es fiel mir an ihnen auf das längere Festhalten an einfachen mehr kindlichen und mechanischen Spielen. Sie zeigten nicht den in dieser Zeit einsetzenden Drang nach Selbständigkeit, fühlten sich vielmehr am wohisten, wenn ihnen alle eigene Verantwortung für ihr Tun abgenommen war. Es fehlte ferner das der Pubertätszeit eigene gesteigerte Selbstgefühl mit der Neigung zu Flegeleien, die gesteigerte Sentimentalität, der gesteigerte Tatendrang, der zum Ausbau allerlei phantastischer Pläne führt. 
Es wäre sehr interessant, festzustellen, ob schon einmal ein Eunuchoider an Dementia praecox erkrankte. In der Literatur konnte ich keine dahingehenden Anhaltspunkte finden.

Die Intelligenz machte dem Alter und der Ausbildung entsprechende normale Fortschritte, und es machte sich eine Neigung zum Kritisieren und Nörgeln bemerkbar.

Eine sichere Leistung der geschlechtlichen Reifung ist in den psychischen Funktionen die Ausbildung des cerebralen Geschlechtstriebes; dies lehren uns die Ausfallserscheinungen. Ohne Frage macht der cerebrale Geschlechtstrieb mit seinen Folgen den Menschen erst zu einem vollwertig männlichen oder weiblichen Individuum. Er hat einen bedeutenden Einfluß auf die ganze psychische Persönlichkeit. Dem Eunuchoiden fehlt im sexuellen Triebleben etwas Wesentliches für sein psychisches Erleben, ein ausschlaggebender Faktor für das Entstehen, die Färbung und die Richtung sozialer und anderer altruistischer Gefühle, es sei nur an die Bedeutung der Liebe zu Gattin und Kinder und an den Familienbegriff mit seinen sozialen Folgerungen erinnert. Es fehlt damit auch beim Eunuchoiden der Einfluß entsprechender Werturteile auf sein Handeln, es fehlt die Leitung der Persönlichkeit durch das Geschlechtsgefühl.

Weiterhin zeigt sich der Einfluß der Geschlechtsfunktion auf die Psyche beim Weibe. Psychische Schwankungen begleiten die Menstruationszeiten. Die Einwirkung der verschiedenen Phasen des Fortpflanzungsgeschäftes auf die weibliche Psyche, der Unterschied zwischen der Mutter und der alten Jungfer ist hinreichend bekannt. Die Kenntnis dieser angeführten Tatsachen hatte dazu geführt, daß man sich lange Zeit sehr eingehend mit den Beziehungen zwischen Genitalfunktion resp. Genitalleiden und Nervenleiden befaßte und den Genitalleiden einen allzu großen Einfluß auf die Nervenleiden einräumte. Man schrieb den Perversitäten des Geschlechtstriebes und rein funktionellen Anomalien eine überwertige Bedeutung zu, man erklärte hierdurch die Entstehung von Neurosen, ja man schlug ernsthaft vor, Geisteskranke durch gynäkologische Operationen heilen zu wollen. Man suchte alles psychische Geschehen von der Sexualsphäre abhängig zu machen. So hat leider die Erkenntnis der Bedeutung der inneren Sekretion der Geschlechtsdrüse eine Menge Auswüchse gezeitigt und diese in Mißkredit gebracht. Kastrationen wegen psychischer Sexualleiden waren nicht so selten.

Kraft - Ebing beschreibt einen sehr lehrreichen Fall. Es wurde gegen seinen Rat die Kastration bei einem „ex masturbatione schweren neurasthenischen“ jungen Manne vorgenommen. Die gewünschte Beruhigung der Genitalsphäre blieb zunächst aus, trat erst nach 2 Jahren ein (wie ja die Libido immer erst nach längerer Zeit schwindet). Der Autor schreibt weiter, das Leiden hatte offenbar nicht seinen einzigen Grund in der Masturbation bzw. Erkrankung der Sexualsphäre und gelangte 
nicht zur Heilung, als Masturbation und Pollutionen endlich auf ein Minimum reduziert waren.

In der Literatur finden sich mehrfach Angaben über psychische Veränderungen bei Eunuchoidismus. Von den eunuchoiden Riesen wird berichtet, da 3 sie geistig minderwertig seien.

Von seinen Späteunuchoiden berichtet Falta ${ }^{1}$ ), daß Angaben über Veränderungen des moralischen und psychischen Verhaltens sehr häufig seien, daß sie psychisch leichter erregbar geworden seien und zum Jähzorn und zur Lügenhaftigkeit hinneigen. Wenn Falta schreibt, das Gefühl der fehlenden Manneskraft führt hier oft zur Schüchternheit, Verschlossenheit, eventuell zu tieferen seelischen Depressionen, so erscheint mir eine derartige psychogene Genese der Erscheinungen nicht richtig. Zudem wissen wir ja, daß das verschlossene, zurückgezogene und stille Verhalten auch den Früheunuchoiden ja schon in deren Kindheit eigen ist. Dem Früheunuchoiden nun können wir nicht gut ein Gefühl oder das Bewußtsein der fehlenden Manneskraft unterschieben, da er ja niemals eines besessen hat. Der Eunuchoide wird weiter als kindlich beschrieben,' das Wort Infantilismus spielt eine Rolle. So spricht z. B. Peritz ${ }^{2}$ ) von einem eunuchoiden Infantilismus. Er will sogar zur Differentialdiagnose gegen die hypophysäre Fettsucht das kindliche Verhalten der Eunuchoiden verwenden können, das bei Erkrankungen an hypophysärer Fettsucht fehle.

Ausführlich behandelt Sterling ${ }^{3}$ ) in seiner Arbeit den Charakter der Eunuchoiden. Wenn Sterling meint, daß das Krankheitsbild des Eunuchoidismus selten sei, so trifft das wohl nur für die ausgesprochenen Fälle zu, die Ubergangs- und die leichteren Formen sind nach meiner Erfahrung gar nicht so selten. Ich habe den Eindruck, daß Sterling bei der Diagnose des Eunuchoidismus den körperlichen Symptomen nicht immer genügend Rechnung getragen hat. Einige seiner Fälle sind nach den somatischen Symptomen meiner Ansicht nach kein reiner Eunuchoidismus.

Sterlings zweiter Fall ist ein 13 jähriger Knabe. Beginn 2 Jahre vorher mit ausgesprochener Adipositas, Polydipsie, die Augenbrauen sind fast total von Haaren entblößt, Wimpern an den oberen Lidern dürftig, cariöse Prozesse an den Zähnen, die Pupillen sind mäßig weit, reagieren gar nicht a uf Lichteinfall und Konvergenz. Die Sehnenreflexe sind ziemlich schwach. Nasaler Beiklang der Sprache, für welchen keine genügende Erklärung zu finden ist. Das alles sind Symptome, die die Zugehörigkeit des Falles zum reinen Eunuchoidismus zweifelhaft erscheinen lassen. Die Pupillenstarre, schwachen Sehnenreflexe und nasale Sprache lassen an einen beginnenden cerebralen Prozeß denken, so daß der Hypogenitalismus sekundärer Natur sein könnte. Der dritte Fall ist eine 57 jährige Frau.

1) Falta, „Die Erkrankungen der Blutdrüsen“. 1913.

2) Peritz, Neurol. Centralbl. 1910; Monatsschr. f. Psych. 1913.

3) Sterling, Klin. Studien über den Eunuchoidismus. Zeitschr. f. d. ges. Neur. u. Psych. 1913. 
Mit 17 Jahren Menses, mit 18 Jahren Ehe, 7 Kinder, mit 48 Jahren Menopause, mit deren Beginn Fettleibigkeit, seit 6-7 Jahren geringer Grad von Urininkontinenz. Auch dieser Fall hat mich nicht überzeugt. Ebenso scheint mir Fall 4 zweifelhaft: 50 jähriger Mann, Geschlechtstrieb mit 17 Jahren, Ehè mit 22 Jahren. 5-6 mal täglicher Geschlechtsverkehr und während der Menstruation der Frau Onanie. Zwei Kinder. Mit 32 Jahren Nachlassen der Libido, mit 35 Jahren impotent. Bis zum 3\%. Lebensjahr eher mager, dann fettleibig. Auf der Abbildung lassen sich keine eunuchoiden Skelettmerkmale erkennen. Die Arme erscheinen eher kurz. Es könnte sich also höchstens um einen Späteunuchoiden handeln. Die psychische Entwicklung ließ seit der Kindheit viel zu wünschen ubrig, er ließ sich immer von seiner Frau unterhalten. Wir können also in diesem Falle die psychische Veränderung, die schon vor dem Beginn des Eunuchoidismus bestanden hat, nicht mit diesem in Zusammenhang bringen. Eigentümlicherweise fuhrt Sterling in diesem Fall den psychischen Zustand mit zur Sicherstellung der Diagnose an. Auch dieser Fall zeigte mangelnde Augenbrauen und cariose Zähne.

Fall 5 ist ein 56 jähriger Mann mit ausgesprochener lebhafter Libido seit dem 16. Lebensjahr bei absoluter Impotenz. Mit $18 \mathrm{Jahren}$ Ehe, zur Zeit noch Geschlechtstrieb. Adipositas seit 4-5 Jahren. Bei Fall 7, einem 14 jährigen Knaben, findet sich eine Wachstumshemmung. Fall 9 läßt eher an eine konstitutionelle Dystrophia adiposo-genitalis denken. Die Sella turcica zeigte eine deutliche Verengerung. Der Knabe ist ,gut gebaut". Jedenfalls scheint der Fall kein reiner Eunuchoidismis zu sein. Sterling sagt von ihm, ,in unserem Fall war außer Hypophyse und dem Hoden wahrscheinlich auch die Thyreoidea betroffen". Fall 10. Geschlechtstrieb seit dem 14. bis 15. Lebensjahr. Ehe, 1 Kind, ,absolut normale sexuelle Funktion". Um einen besonders hochgradigen Eunuchoidismus kann es sich also hier kaum gehandelt haben. Wenn Sterling schreibt, als Komplikationen sind die abortiven Formen der Basedowschen Krankheit, Skrofulose, Tuberkulose, Granulose der Lymphdrüse, Migräne, zu erwähnen, so ist dem entgegenzuhalten. daß ich außer der Migräne bei einem verhältnismäßig großen Material von 16 Fällen nicht eine einzige von den anderen als Komplikation angefuhrten Krankheiten sah. Die wichtigste Komplikation des Eunuchoidismus ist nach Sterling die Epilepsie, die in Form von Krampfanfällen, Absences, psychischen Äquivalenten, Ohnmachtsanfällen, rudimentären Zügen des epileptischen Charakters und als Affektepilepsie auftritt. Er unterscheidet drei psychische Typen des Eunuchoidismus.

I. Solche, die intellektuell wenig von dem Durchschnitt abweichen, mit geselligem Charakter, altruistischen Neigungen, Resignation dem sexuellen Defekt gegenüber, leichten Depressionszuständen und im kindlichen Alter Passivität und Trägheit.

2. Imbezille mit geringem Vorstellungsvermögen und Defekte höherer psychischer Leistungen bei emotioneller Abstumpfung.

3. Solche, die bei verhältnismäßig gut erhaltenen höheren psychischen Leistungen uber einen sehr beschränkten Vorstellungs- und Begriffsschatz verfügen und durch absolute Lebensuntüchtigkeit charakterisiert sind (Parasitentypus). idismus.

Außerdem spricht $\mathbf{S}$ terling von einem epileptischen Typus des Eunucho-

Ich halte eine Einteilung in verschiedene Gruppen auf Grund der Psychopathologie. des Eunuchoidismus für unbegründet. Es wäre ja auch verwunderlich, daß ein in den körperlichen Symptomen so gut charakterisiertes Krankheitsbild wie der Eunuchoidismus drei völlig voneinander abzugrenzende Typen auf psychopathologischem Gebiete 
bieten sollte. Die Eunuchoiden zeigen keine qualitative, sondern nur quantitative Differenzen in den Symptomen. Die Intelligenz aber ist in die qualitativen Unterschiede außerhalb normaler Grenzen nicht einbezogen. Ein Intelligenzdefekt gehört nicht zu den Symptomen des Eunuchoidismus.

Wenn man wiederholt in der Literatur die Ansicht vertreten liest. da $\beta$ die Eunuchoiden bestrebt sind, ihre sexuellen Defekte zu verdecken, oder absichtlich das Thema zu vermeiden suchen, so mag das bei Späteunuchoiden resp. Spätkastraten der Fall sein. Nur diese können ja auch ein völliges Bewußtsein ihres erworbenen Defektes haben. Dagegen bin ich nach meiner Erfahrung bei den Früheunuchoiden der Ansicht, daß die Vorstellung von dem vorhandenen Defekt natürlich eine sehr minimale und oberflächliche ist. Es ist wohl verständlich, daß .jemand, der niemals erotisch empfunden hat, kein Bewußtsein vom erotischen Empfinden oder dem Defekte eines solchen haben kann. Ich habe bei meinen Kranken die Erfahrung gemacht, daß diese bei Fragen über die vorhandenen Defekte, soweit sie eben den Sinn der Fragen erfassen konnten, nicht mehr Widerstand zeigten als bei anderen Fragen. Sie sind überhaupt schwer zugänglich. Wenn man die Patienten aber vor Scheinprobleme stellte und etwas aus ihnen heraus- resp. hineinfragen wollte, was ihnen, eben wegen ihres vorhandenen angeborenen Defektes zu beantworten unmöglich war, wurden sie natürlich ablehnend und auch gereizt. Allgemein macht man die Erfahrung, daß Früheunuchoide den Defekt selbst nicht hoch bewerten, daß ihre Vorstellung vom Sexualleben und dessen Bedeutung, die sich z. B. Gebildete durch Literatur und Befragen verschaffen, immer nur das Oberflächliche streift und streifen kann. Denn vón den Gefühlsqualitäten und den Gefühlswerten, die ihnen fehlen, kann ihnen auch die beste Literatur nicht zum Bewußtsein verhelfen. Utber diese Frage erhielt ich besonders von einem Kollegen mit ausgesprochenem eunuchoidem Hochwuchs interessante Aufschlüsse.

Sänge $\mathbf{r}^{1}$ ) berichtet von 2 Fällen von Präpubertätseunuchoidismus, einem $13^{1} / 2$ jährigen Knaben, bei dem sich seit $1 \frac{1}{2}$ Jahren eine auffallende Fettsucht entwickelt hatte, und gleichzeitig eine Wesensänderung einsetzte. Er wurde jähzornig, ließ in seinen Schularbeiten nach. Der zweite Fall, ein 14jähriger Knabe mit Fettsucht, kleinem Penis und Kryptorchismus zeigte ähnliche psychische Veränderungen.

Wenn ich jetzt an die Betrachtung der Psychopathologie des Eunuchoidismus gehe, betone ich ausdrücklich, daß ich zunächst nur solche Fälle herangezogen habe, deren Diagnose nach den somatischen Symptomen absolut einwandfrei ist. Insbesondere ist dies der Fall bei hoch-

1) Sänger, Deutsche med. Wochenschr. 1910; Sitzungsbericht, Zeitschr. f. Nervenheilk. 1914. 
wüchsigem Eunuchoidismus. Fälle mit sichtbarer Beimengung anderer Drüsen, der Hypophyse und Bchilddrüse, habe ich in dieser Arbeit ganz ausgeschaltet. Diese bedürfen einer getrennten Bearbeitung.

Die intellektuellen Fähigkeiten der Eunuchoiden liegen in normalen Grenzen. Nach meinen Erfahrungen gehört ein Intelligenzdefekt nicht zu den Symptomen des reinen Eunuchoidismus. Werden solche Fälle beobachtet, so muß man eingehend prüfen, ob nicht eine Kombination mit Schwachsinn anderer Ätiologie vorliegt. Es besteht die Möglichkeit, daß bei hochgradigen epileptischen Symptomen ein Schwachsinn späterhin erworben wird, doch müssen solche Fälle wohl sehr selten sein.

Die Fähigkeiten sind vorhanden; anders aber verhält es sich mit der Ausbildung der Intelligenz und den erworbenen Kenntnissen. Von den Kenntnissen kann man sagen, daß sie günstigstenfalls nicht den Durchschnitt dessen übersteigen, was man von einem Menschen gleicher sozialer Stellung und beruflicher Tätigkeit verlangen muß. Hiervon machten auch die beiden akademisch gebildeten Eunuchoiden, die ich beobachten konnte, keine nennenswerte Ausnahme; es handelte sich um einen Theologen und einen Arzt. Letzterer war in einem ganz kleinen Spezialfach tätig. Die Beschäftigung mit belehrender Literatur ging nicht über die notwendige Fachliteratur hinaus. Bei gebildeten Eunuchoiden findet man häufig eine Beschäftigung mit religiösen Fragen unter Bevorzugung des Mystizismus.

Der Arbeitstrieb läßt sofort nach, sobald die direkte Arbeitsnotwendigkeit fortfällt und die rein persönliche Anteilnahme, die durch Examen, Erwerb des Lebensunterhaltes und ähnliche Momente gegeben ist, durch Erreichung des Notwendigen sich erschöpft hat. Sie zeigen keinen Fleiß, aus Neigung sich nützlich zu machen und zu helfen, sondern nur Beschäftigungsneigung, soweit ihre egoistischen Bedürfnisse dieses erfordern und als Reiz wirksam sind. Die Eunuchoiden können wohl auch Fleiß entwickeln, wenn die Notwendigkeit es mit sich bringt, aber es ist ein Fleiß ohne Arbeitsfreudigkeit. Hierdurch unterscheiden sich die Eunuchoiden ganz wesentlich von den Epileptikern.

Ich möchte sagen, der Defekt ist kein Intelligenzdefekt, sondern ein Defekt des Triebes zur Ausbildung und der`Initiative zur Verwertung der Intelligenz.

Von dem Charakter der Eunuchoiden kann man ganz allgemein sagen, daß er ein passiver, negativer ist, womit nur die gelegentlich explosiv und aktiv werdende Reizbarkeit kontrastiert. Es besteht zweifellos eine gewisse Ähnlichkeit zwischen dem Charakter der Eunuchoiden und der Epileptiker, aber im wesentlichen nur in den krankhaften Zügen. Gemeinsam ist beiden die egozentrische Einengung, 
Schwerfälligkeit, Empfindlichkeit, Reizbarkeit, Explosivität, außerdem die Frömmigkeit und in einigen Fällen auch eine gewisse Pedanterie. Auch Gutmütigkeit zeigen manche Eunuchoiden, die aber eher passiv duldet, als aktiv sich betätigt, und gewissermaßen mehr einen Mangel an Reaktion darstellt. Der wesentliche Unterschied gegenübier dem epileptischen Charakter liegt praktisch darin, daß dem Eunuchoiden die sozial bedeutungsvolle Aktivität der Epileptiker fehlt.

Es ist hinreichend bekannt, daß Intelligenz und soziale Eigenschaften durchaus nicht ineinandergreifen resp. parallel gehen. Epileptiker, selbst mit schweren Defekten, können in einer ihnen angepaßten Umgebung immer noch durch ihre sozial wichtigen Charakter: züge brauchbar sein. Sie haben den Drang, sich eine nutzbringende Beschäftigung zu suchen, haben Freude an der Arbeit und dem Erfolg, sie zeigen Teilnahme und Mitgefühl gegen ihre Umgebung, sind höflich, entgegenkommend, mitteilsam, sie haben das Bedürfnis sich anzuschließen. Der epileptische Charakter ist der sozial wertvollere aktive, der eunuchoide der passive asoziale Charakter. Demnach kann von einer Kombination mit psychischer Epilepsie oder von einem epileptischen Charakter des Eunuchoidismus nach meiner Ansicht keine Rede sein.

In den Anamnesen fand ich über die Eigenschaften der Eunuchoiden in der Kindheit folgende Angaben: Sie sind still, schüchtern, folgsam, ängstlich, unselbständig, schwer zugänglich, wenig anhänglich und gern für sich allein, schließen sich schwer und ungern an andere Kinder an. Ende der zweiten Kindheitsperiode werden sie als denkfaul, empfindlich, reizbar, launenhaft, resistent, $\cdot$ jähzornig geschildert, sie zeigen wenig Ehrgeiz und Phantasie beim Spiel.

Von einer ausgesprochenen Wesensänderung in den Jahren, in denen normalerweise die Pubertät mit ihren bekannten Erscheinungen auf psychischem Gebiete einsetzt, habe ich nichts erfahren. Nur scheinen um diese Zeit die epileptoiden Symptome, die Empfindlichkeit und der Jähzorn zuzunehmen, sie werden unzufrieden, nörgelnd, fauler, klagen viel über Kopfschmerzen. Auch scheint um diese Zeit der Hang zur Frömmigkeit mehr hervorzutreten. Erst einige Zeit später treten die ersten gröberen epileptischen resp. epileptoiden Krankheitsmerkmale (Migräne, Schwindelanfälle usw.) hervor.

Von den erwachsenen Eunuchoiden läßt sich im allgemeinen folgendes sagen: Ausdrucksbewegungen und Mimik sind ohne Modulationsfähigkeit, haben etwas Schwerfälliges, Müdes und Apathisches, der Gesichtsausdruck zeigt in Andeutung eine gewisse, allen gemeinsame Ähnlichkeit, die insbesondere auf der Faltenbildung um Mund und Augen zurückzuführen ist. Neben dieser Ausprägung des eunuchoiden Types sind die Kranken indifferent, ohne individuelle Persönlichkeits- 
färbung. Sie zeigen eine Einengung des Gefühlslebens und eine Verflachung bestimmter Gefühlswerte, die für solche Zielvorstellungen richtunggebend sind, die zur Ausbildung und Verwertung der vorhandenen intellektuellen Fähigkeiten führen. Ferner fehlen Gefühlswerte, die aus dem sexuellen Triebleben erwachsen und normalerweise zu sozial wichtigen altruistischen Folgerungen führen, zum Verantwortlichkeitsgefühl gegenüber Staat und Familie und dem sozial wichtigen Trieb zum gemeinsamen Leben. Die Folge ist eine ausgesprochene Egozentrizität und Indifferenz, die Teilnahmlosigkeit gegen die nähere und weitere Umgebung, die Abgeschlossenheit, das mangelnde $\mathrm{Be}-$ dürfnis nach Anschluß und Freundschaft, die mangelnde Aufnahmefähigkeit und Gefühlsbetonung bestimmter Erlebnisse. Aus dem Mangel an Initiative, an Entschlußfähigkeit, Selbständigkeit, Arbeitsfreudigkeit und Ehrgeiz können wir andererseits wieder auf den Mangel an positiven, der Persönlichkeit richtunggebenden Gefühlswerten schließen. Diese geschilderten Eigenschaften haben sicher nichts mit dem epileptischen Charakter gemeinsam. Von allen diesen negativen Eigenschaften finden wir im epileptischen Charakter gewissermaßen das Positiv.

Versuch einer Gegenüberstellung des epileptischen und eunuchoiden Charakters.

Epileptischer Charakter:

Aktiver Charakter:

Regsam,

lebhaft,

mitteilsam,

zutunlich,

entgegenkommend, höflich,

brav, bieder,

anhänglich,

Teilnahme, Mitgefühl, Bedürfnis nach Anschluß,

Gewissenhaftigkeit,

interessiert,

Selbstbewußtsein,
Eunuchoider Charakter:

Passiver Charakter:

Initiativelos, apathisch, still, zurückgezogen, verschlossen, schwer zugänglich, abweisend, mißtrauisch, resistent, wenig anhänglich, Teilnahmlosigkeit gegen nähere $u$. weitere Umgebung, keine Neigung zu Anschluß und Freundschaft,

Mangel an Verantwortlichkeitsgefühl,

mangelnde Ausbildung und Verwertung der Intelligenz, geringe Aufnahmefähigkeit und Gefühlsbetonung,

Unselbständigkeit und Entschlußunfähigkeit, 
Ehrgeiz,

Beschäftigungstrieb und Arbeitsfreudigkeit, positiv, sozial wichtig,
Mangel an Ehrgeiz, Arbeitswille nur bis zur Erschöpfung der Arbeitsnotwendigkeit. negativ, asozial.

Beiden gemeinsam:

Schwerfällig, verlangsamt, egozentrische Einengung, Reizbarkeit und Explosivität, Empfindlichkeit, Pedanterie und Frömmigkeit.

An epileptischen psychotischen Merkmalen finden sich in den Entwicklungsjahren immer Kopfschmerzen. Häufig sind Migräne und kurz dauernde Schwindelanfälle. Die Migräneanfälle sind zum Teil sehr schwer (z. B. Fall 3, mit dem Gefühl des schemenhaft Unwirklichen für sich und die Umgebung). Verstimmungen mit gesteigerter Reizbarkeit, Ängstlichkeit und Hemmung. Alkoholintoleranz mit pathologischen Räuschen und Vagabundieren beobachtete ich in einem Falle, ferner in Fall 4 einen 20stündigen Schlafzustand und in Fall 5 einen epileptiformen Anfall und einen kollapsartigen Zustand.

Auf jeden Fall ist der eunuchoide Charakter ein wohl umschriebener und selbständiger. Auffallen muß, daß die krankhaften epileptischen Charakterzüge auch dem Eunuchoidismus eigen sind und die psychotischen Merkmale durchaus dieselben sein können, während andererseits sich die Charaktereigenschaften des Epileptikers beim Eunuchoidismus im Negativ finden. Man kann nach meiner Ansicht hieraus folgende Schlüsse ziehen: Einmal muß es noch eine andere Schädigung bzw. konstitutionelle Störung im Körper geben, welche bei Erhaltensein der Geschlechtsdrüsenfunktion zu derselben Verschiebung im Gleichgewicht bestimmter innersekretorischen Drüsen führt. Tritt diese Schädigung bzw. Konstitutionsstörung bei erhaltener Geschlechtsdrüsenfunktion ein, so ist die echte epileptische Konstitution bzw. die Krankheit Epilepsie gegeben. Zweitens meine ich aus dem Unterschied im eunuchoiden und epileptischen Charakter entnehmen zu können, daß die asozialen negativen Züge des eunuchoiden Charakters voll und ganz dem Geschlechtsdrüsenausfall und dessen sekundären Folgen zuzuschreiben sind.

Jedenfalls hat uns das Studium des Eunuchoidismus erneut einen Beweis dafür erbracht, daß die Tätigkeit der Blutdrüsen auf die Entwicklung und Funktion des Zentralnervensystems einen gesetzmäßigen und entscheidenden Einfluß ausübt. Einer pathologischen Korrelation im Blutdrüsensystem entspricht hier gesetzmäßig ein bestimmter Funktionszustand des Zentralnervensystems, der sich zum Teil in Symptomen äußert, die wir klinisch als zum Bilde der Epilepsie gehörig kennen. 
Zum Schluß ist hervorzuheben, daß in den nervösen Folgeerscheinungen der Kastration und in den leichteren nervösen und psychischen Symptomen des Eunuchoidismus eine große Ähnlichkeit herrscht. Die vasomotorischen Erscheinungen stehen bei den Folgezuständen der Kastration mehr im Vordergrund als beim Eunuchoidismus. Den Reizzustand nach der Kastration im Bereich des übrigen vegetativen Nervensystems, Brechreiz und nervöses Erbrechen fand ich bei Eunuchoiden in Fall 4 und 5 angedentet. Ferner sei hier nochmals hingewiesen auf die Angaben Pfisters, daß die Operierten häufig über Kopfschmerzen klagen, die klinisch das Bild einer Migräne bieten, und daß häufig von Frauen die Angabe gemacht wurde, sie seien seit der Operation reizbarer geworden. Dann auf die Angaben Hirschfelds, der von einem männlichen 49 jährigen Spätkastraten schreibt, daß dieser erhebliche Affekterregbarkeit zeigte, jetzt sehr zurückgezogen lebte und täglich in die Kirche ging, während Gedächtnis und Intelligenz nicht abgenommen hatten. Bei einem mit 21 Jahren Kastrierten sah Hirschfeld ganz regelmäßige Zeiten, in denen der Operierte an Schwindel, Mattigkeit, Kopfweh, starker Gereiztheit und seelischer Verstimmung litt.

Der Ausfall der inneren Sekretion der Geschlechtsdrüse ist für die Kastrationsfolgen mit Sicherheit das ursprünglichste ätiologische Moment, und nach meiner Ansicht auch für den Eunuchoidismus. Für den Ausfall der Geschlechtsdrüsenentwicklung gibt es zwei Möglichkeiten. Einmal kann die die allgemeine Reifung einleitende Involution der Zirbeldrüse eine unvollständige sein, so daß Reize, die normalerweise die Reifung der Geschlechtsdrüse einleiten, ausfallen, oder aber, und das scheint mir wahrscheinlicher, die unterwertig angelegten Geschlechtsdrüsen sind auf die vorhandenen Reize nicht reaktionsfähig. Die nach Kastration, im Klimakterium und beim Eunuchoidismus krankhaft gesteigerte Reaktionsfähigkeit und Labilität des vasomotorischen und anderer Teile des vegetativen Nervensystems, sowie die bei diesen Zuständen erhöhte Adrenalinempfindlichkeit machen es wahrscheinlich, daß der Ausfall der Geschlechtsdrüse eine gesteigerte Sensibilisierung des Sympathicus zur Folge hat.

Da das Adrenalinsystem bekanntlich entwicklungsgeschichtlich selbst ein Abschnitt des Sympathicus ist, besteht die Möglichkeit, daß auch dieses nach Fortfall der Geschlechtsdrüsenfunktion in seiner Funktion im Sinne einer gesteigerten Reaktionsfähigkeit gestört ist, wofür auch die Hypertrophie der Nebennieren nach Kastration sprechen würde.

Weiter ist die Funktion der Nebennieren für den normalen Ablauf der Muskelarbeit von enormer Wichtigkeit.

Die Chromierbarkeit der Markzellen der Nebennieren nimmt bei forcierter Muskelarbeit, wie sie im Krampfanfall ja im besonderen 
geleistet wird, ab. Diese Tatsache ist experimentell leicht nachzuprüfen. Rössle ${ }^{1}$ ) sah in 3 Fällen von Tod im epileptischen Anfall und nach solchem große fette Nebennieren mit auffällig wenig Mark. $\mathrm{Sch}$ morl berichtet von einem jungen Manne, der nach einer anstrengenden Radrennfahrt plötzlich verstarb; als einziger pathologischer Befund wurde Unchromierbarkeit der Marksubstanz der Nebennieren erhoben.

Nebennierenlose Tiere sind leicht ermüdbar und zu keiner größeren Muskelarbeit fähig. Ich sah bei Versuchen über Amylnitritkrämpfe an Kaninchen, daß die Krampffähigkeit der Tiere mit experimenteller Entfernung größerer Abschnitte der Nebennieren abnimmt. Bei Tieren, denen ich die Nebennieren ganz herausnahm, konnte ich keine Krämpfe mehr auslösen.

Es steht für mich ganz ohne Frage fest, daß die Nebennieren, und zwar nicht nur die Marksubstanz für den Krampfkomplex eine wesentliche Rolle spielten, und zwar für diejenige Komponente des Krampfbedingungskomplexes, die ich die periphere Krampfkomponente nennen möchte. Eigentümlicherweise hat man bisher noch niemals diese bei den Untersuchungen und Beobachtungen berücksichtigt, sondern einseitig immer die zentrale Komponente untersucht. Die Krampfdisposition ist ein Komplex, in dem beide Bedingungen, die periphere und zentrale, mehr oder weniger krampfdisponibel sein können. Bei der Krankheit Epilepsie scheint mir nun, und hierfür finden sich viele Anhaltspunkte, die periphere Komponente von ganz hervorragender Bedeutung zu sein. In dieser peripheren Krampfkomponente spielen die Nebennieren eine große Rolle für die Krampffähigkeit der quergestreiften Muskulatur, sie regulieren gewissermaßen deren Reizschwelle.

Erwähnt sei noch, daß nach Experimenten, die ich zusammen mit J. Fischer ${ }^{2}$ ) ausführte, bei kastrierten Tieren die Krämpfe nach Amylnitritinhalation früher als bei normalen auftreten, und die Tiere nach dem Krampf stärker benommen sind und sich viel langsamer erholen als gesunde.

Weiter will ich auf die Bedeutung der inneren Sekretion für die Ätiologie des Eunuchoidismus und der Epilepsie nicht eingehen, da ich diese Fragen in einer gesonderten Arbeit behandele.

1) Rössle, Wiener med. Wochenschr. 1910, S. 1380.

2) H. u. J. Fischer, Diese Zeitschr. Orig. 22, 241. 1914. 